\title{
Review
}

\author{
Iwona Cicha*, Christoph D. Garlichs and Christoph Alexiou
}

\section{Cardiovascular therapy through nanotechnology - how far are we still from bedside?}

\begin{abstract}
Recent years brought about a widespread interest in the potential applications of nanotechnology for the diagnostics and the therapy of human diseases. With its promise of disease-targeted, patient-tailored treatment and reduced side effects, nanomedicine brings hope for millions of patients suffering of non-communicable diseases such as cancer or cardiovascular disorders. However, the emergence of the complex, multicomponent products based on new technologies poses multiple challenges to successful approval in clinical practice. Regulatory and development considerations, including properties of the components, reproducible manufacturing and appropriate characterization methods, as well as nanodrugs' safety and efficacy are critical for rapid marketing of the new products. This review discusses the recent advances in cardiovascular applications of nanotechnologies and highlights the challenges that must be overcome in order to fill the gap existing between the promising bench trials and the successful bedside applications.
\end{abstract}

Keywords: cardiovascular diseases; cardiovascular drugdelivery nanosystems; clinical trials; diagnostic nanoparticles; nanomedicine.

\footnotetext{
*Corresponding author: Iwona Cicha, PhD, Cardiovascular Nanomedicine Unit, Section of Experimental Oncology and Nanomedicine, University of Erlangen-Nuremberg, Glückstr. 10a, 91054 Erlangen, Germany, Phone: +49 91318543953 , Fax: +49 9131 8534282, E-mail: Iwona_Cicha@yahoo.com Christoph D. Garlichs and Christoph Alexiou: Section of Experimental Oncology and Nanomedicine (SEON), Else KrönerFresenius-Stiftung-Professorship, ENT Department, University Hospital Erlangen, Erlangen, Germany
}

lived with disability, coronary heart disease and stroke rank first and third, respectively (2). Although the clinical management of CVD has improved in Western Europe leading to about 20\% decrease in age-standardized death rates for CVD in the last two decades (1), the number of CV deaths is predicted to increase from 17.1 million worldwide in 2004 to 23.6 million in 2030 due to the increasing impact of obesity and metabolic syndrome. In this paper, we review the potential applications of nanotechnologies in cardiovascular medicine, the current stand of clinical studies and pilot trials, and the obstacles to overcome before the nanomedical approaches can be safely introduced to the clinical practice.

\section{Potential applications of nanotech- nologies in cardiovascular medicine}

Nanomedicine offers a unique platform for novel approaches to the diagnosis and therapy of cardiovascular diseases. The possible applications range from plaque imaging and thrombus detection to the targeted drug-delivery, stent endothelialization and blood vessel regeneration. This subchapter summarizes recent advances in the preclinical experimental efforts to implement nanomedical approaches to cardiovascular disorders.

\section{Nanosystems for detection and characteriza- tion of cardiovascular disorders}

\section{Imaging modalities and nanoprobes}

\section{Introduction}

Cardiovascular diseases (CVD) account for almost 50\% of all deaths in Europe and around 30\% of all deaths worldwide (1). According to the Global Burden of Disease Study 2010, when combining years of life lost and years
The molecular imaging techniques routinely used in cardiovascular medicine are constantly being optimized to better detect atherosclerotic plaques, but none of the modalities is without limitations (3). Whereas magnetic resonance imaging (MRI), a noninvasive and nonionizing imaging technique has an excellent resolution but 
low sensitivity, positron emission tomography (PET) has the highest sensitivity of all imaging modalities and an unlimited penetration depth. These advantages are counterbalanced by its low resolution, very high cost, and radioactivity. Optical fluorescence imaging, suitable for e.g., imaging plaque endothelium, can be difficult to quantify in tissues more than a few millimeters in depth. Thus far, the preclinical studies performed in order to investigate the diagnostic and therapeutic benefit of nanoparticles in atherosclerosis mostly utilized the MRI contrast agents, which consist of suspended colloids of nanoparticles and, when injected during imaging, reduce the $\mathrm{T}_{2}$ signals of absorbing tissues. In particular, either paramagnetic gadolinium chelates or iron oxide-based contrast agents, such as superparamagnetic iron oxide (SPIO, particle size of 120-180 nm) and ultrasmall superparamagnetic iron oxide (USPIO, $60 \mathrm{~nm}$ particle size) have been used. Recently, multi-modal contrast agents or imaging probes detectable with multiple molecular imaging techniques have emerged, which promise a better sensitivity and accuracy of atherosclerotic plaque detection and classification $(4,5)$.

\section{Detection of atherosclerotic lesions}

Early identification and aggressive pharmaceutical and/ or interventional treatment of atherosclerotic plaques can help to reduce the incidence of acute ischemic events. Conjugating nanoparticles to specific ligands that target endothelial cell adhesion molecules [vascular cell adhesion molecule-1 (VCAM)-1 $(6,7)$, as well as E- and P-selectins $(8,9)]$ has proven a successful experimental approach to noninvasive in vivo imaging of the early stages of atherosclerosis. Imaging nanoparticles have also been implemented for vulnerable plaque identification based on their ability to detect intraplaque macrophages, lipids, angiogenesis, apoptosis, or thrombotic deposits [reviewed in detail in (10)]. Below, several approaches to atherosclerotic plaque characterization are briefly outlined. Macrophage burden in atherosclerotic plaques in vivo can be estimated using unlabeled superparamagnetic iron oxide nanoparticle (SPION), as shown in hyperlipidemic rabbits by differential phase optical coherence tomography (OCT) (11) and MRI $(12,13)$ as well as in atherosclerotic plaques of ApoE-deficient mice (14). However, as the uptake of SPION is not specific to the plaque macrophages (15), targeting of macrophages [e.g., with homing pepride LyP-1 $(16,17)]$ and macrophage receptors [e.g., CD36-targeting gadolinium-containing liposomes $(18,19)$ and scavenger receptor-A-targeting iron oxide nanoparticles (20)] is helpful to enhance the labeling efficiency of the particles in vivo. Lipoproteins, natural nanoparticles of 5-20 nm diameter, represent good candidates for the transfer of imaging nanoparticles into the lesions. High-density lipoproteins (HDL)like nanoparticles enriched with gadolinium have been reported to specifically image plaques in vivo $(21,22)$. Detection of vulnerable plaques in mice was also facilitated by anti-mouse OxLDL polyclonal antibodies conjugated to USPIO (23).

Imaging apoptotic cells is another possible approach to identifying plaques with vulnerable morphology, as shown by studies utilizing phosphatidylserine-targeting peptides linked to USPIO (24), as well as annexin A5-labeled SPION (25) and gadolinium nanoparticles (26). Neoangiogenesis, a common feature of advanced vulnerable plaques can also constitute an MRI detection target for plaque characterization, as shown in atherosclerotic rabbits (27) administered gadolinium-containing perfluorocarbon nanoparticles targeted to $\alpha_{v} \beta_{3}$-integrin, one of the key mediators of neovessel formation $(28,29)$.

Thrombus formation that occurs on the luminal surface of atherosclerotic plaques presents yet another target for detection by MRI. For this purpose, anti-fibrin antibodies conjugated to gadolinium-diethylene triamine pentaacetic acid (DTPA)-perfluorocarbon nanoparticles were successfully used (30), as well as the fibrin-targeting CREKA peptide-bound lipopeptide nanoparticles (31), or a commercially available gadolinium-linked fibrin-binding peptide EP-2104R (32). These studies indicate that employing targeted nanoparticles to refine the available non-invasive techniques should not only advance the detection of vulnerable plaques but also enable monitoring the disease progression and therefore improve the risk stratification.

\section{Detection of high-risk aneurysms}

Abdominal aortic aneurysms (AAA) occur in 5\% to 9\% of the population over the age of 65 years and are the tenth leading cause of death in Western countries (33). Several recent reports show the feasibility of nanotechnological approach to in vivo aneurysm detection and characterization using MRI and near-infrared fluorescence imaging (NIRF). As an example, SPION-enhanced MRI was applied to detect early AAA in ApoE-deficient mice (34). SPION uptake and abdominal aortic diameter were found to correspond to the numbers 
of iron-laden macrophages in the aneurysm. Another approach was tested by Klink et al. (35), who showed that intravenous administration of gadolinium-based fluorescent micellar nanoparticles functionalized with a collagen-binding protein resulted in a significantly higher magnetic resonance signal enhancement in the aneurysmal wall compared with nonspecific micelles. High-resolution MRI allowed longitudinal monitoring of the AAA progression and the increase of the aortic diameter, enabling the discrimination between stable and rupture-prone aneurysms. Nearly in parallel, a study by Kitagawa et al. evaluated NIRF imaging of AAA using Arg-Gly-Asp (RGD)-conjugated human ferritin nanoparticles labeled with Cy5.5. In a mouse model of AAA, a significantly higher signal in AAA relative to non-diseased regions was obtained using RGD-ferritin nanoparticles than with unconjugated nanoparticles (36). These studies suggest that targeting nanoparticles allow more comprehensive characterization and prognosis of aneurysmal disease.

\section{Imaging inflammation after cerebral or cardiac ischemia}

Vascular damage and inflammation critically affect patients' outcomes after stroke. Nanoparticle-based contrast agents can be applied to characterize the extent of inflammation, as demonstrated by several studies in an experimental murine model of cerebral ischemia. In those early studies (2001-2004) by Rausch et al. (37, 38), Kleinschnitz et al. (39), and Schroeter et al. (40), SPION have been utilized for MR imaging of the brain inflammation after stroke and showed macrophage-specific accumulation in the infarcted brain region. The ability of USPIO-enhanced MRI to detect inflammatory response surrounding the ischemic regions has subsequently been confirmed by Wiart et al. (41). Most recently, Frechou et al. (42) applied USPIO conjugated to VCAM-1-targeting peptide in order to detect cerebral expression of VCAM-1 after experimental stroke in mice. The study showed that such targeted MRI contrast agent can be useful for characterizing the vascular damage associated with cerebral ischemia.

Following myocardial infarction (MI), uncontrolled inflammation and adverse cardiac remodeling can ultimately result in heart failure. Thus far, very small iron oxide nanoparticles (43) and micrometer-sized iron oxide particles (44) have been tested to assess inflammation in murine models of MI showing their ability to characterize the course of pathologic process after myocardial ischemia.

\section{Nanosystems for diagnosis of cardiovascular disorders}

Nanotechnology-based signal amplification for biosensing has been a rapidly developing field. Several categories of nanomaterials such as gold nanoparticles, magnetic oxide nanoparticles, or quantum dots have multiple potential applications in this important aspect of cardiovascular diagnostics. This subchapter focuses on the application of nanosystems for the estimation of disease biomarkers.

\section{Estimating the burden of thrombosis}

Intravascular thrombosis, the formation of life-threatening obstructive blood clots within the vessels, underlies a number of cardiovascular disorders such as heart attack, ischemic stroke, pulmonary embolism, and deep vein thrombosis $(45,46)$. Within the coagulation cascade, thrombin is the most important serine protease (47), but the diagnostic tests are lacking that directly reflect its activity in clinical settings. Recently, Lin et al. described the development of novel urinary nanomarker assay based on thrombin-sensitive iron oxide nanoparticles that allows detection of thrombin activity in vivo (48). The nanomarkers were produced by coupling iron oxide nanoworms with thrombin-cleavable peptides linked to a synthetic reporter system, composed of protease-resistant peptide, glutamate-fibrinopeptide $B$, which was modified at the termini with ligands detectable by an immunoassay (fluorescein, or Alexa488, and biotin). In a mouse model of pulmonary embolism induced by thromboplastin (49), the authors showed that the circulating nanomarkers could access the local sites of thrombosis and release the reporters, the urinary clearance of which was detectable by ELISA with high sensitivity and significantly correlated with the disease burden as estimated by the histochemically analyzed amount of fibrin deposited in the lungs (48).

The imaging approach to thrombosis detection using microCT has been recently tested in a mouse model of carotid thrombosis using glycol chitosan (GC)-gold nanoparticles. The study showed that these nanoparticles allowed both the detection of primary and recurrent thrombi, and the monitoring the therapeutic efficacy of thrombolysis with tissue plasminogen activator (tPA). Due to a long circulating half-life, GC-gold nanoparticles remained available for entrapment into fibrin matrix for up to 3 weeks, allowing repetition or ongoing monitoring of thrombogenesis and thrombolysis (50). Given the need of rapid and reliable in vivo assessment of the thrombotic 
risk in patients with cardiovascular diseases in order to improve the diagnosis, risk stratification, and management of thrombotic syndromes, those systems represent a very attractive platform for use in clinical practice.

\section{Diagnosis of acute coronary syndromes}

Molecular biomarkers are used as objective indicators of myocardial injury. About $30 \%$ of patients with non-STelevation acute coronary syndrome present without evidence of myocardial necrosis using available assays for cardiac troponin, the biomarker of choice for the serologic diagnosis of acute coronary syndromes. More sensitive assays for troponin are urgently needed to enable an earlier detection of MI and identify patients who are at risk of short-term major adverse cardiac events. Nanotechnology offers several solutions to the drawbacks of the existing cardiac biomarker assays. One of them was recently reported by Cowles and Zhu (51), who applied the dual signal amplification method for the measurement of cardiac troponin I (cTnI) in human serum. The technique consists of sandwich-ELISA, in which detection antibodies are linked by biotin-avidin complex to semiconductor nanoparticle labels (quantum dots) of zinc sulfide. By lowering $\mathrm{pH}$, the release of zinc ions is induced (first step of signal amplification), which act as co-factors for carbonic anhydrase and, at normalized $\mathrm{pH}$, lead to a concentrationdependent activation of this enzyme. Upon addition of substrate, fluorescein diacetate, enzyme activity produces fluorescent product (second step of signal amplification) the concentration of which is measured spectrophotometrically. Using this technique, cTn1 assay was developed and tested on human serum samples, showing superior detection resolution and simple handling (51).

In a study by Ling et al. (52), magnetic resonance relaxometry was used to noninvasively monitor changes in the relaxation properties of antibody-coated magnetic particles when they aggregate upon exposure to a biomarker of interest. As the single-point measurements often do not reflect the directions of the underlying pathologic process, thus hindering diagnostic and prognostic decisions, the authors of this innovative method applied implantable devices containing sensors of three clinically relevant cardiac biomarkers: cTnI, creatinine kinase and myoglobin, to continuously monitor biomarker levels for up to $72 \mathrm{~h}$, with a detection level as low as the $\mathrm{pg} / \mathrm{mL}$ range. In a mouse model of $\mathrm{MI}$, the detected biomarker levels and changes over time differed between experimental and control groups and correlated with infarct size.
These studies underscore the enormous potential of nanotechnologies for improved biomarker detection and thus patients' diagnosis.

\section{Nanosystems for monitoring the treatment efficacy}

Nanoparticle-based imaging may serve not only as a biomarker to identify vulnerable lesions, angiogenesis, or ischemic regions, but can also provide a tool to monitor the therapeutic effectiveness of medication. In a study by Morishige et al. (13), SPION-enhanced MRI was used to monitor the effects of rosuvastatin in hypercholesterolemic rabbits. A recent study by Sigovan et al., applied a similar approach using USPIO to noninvasively monitor the therapeutic effect of irbesartan therapy on macrophage burden in atherosclerotic plaques of ApoEdeficient mice (14). Serial USPIO-enhanced MRI scans were furthermore utilized to monitor the therapeutic effects of an anti-inflammatory drug minocycline in a mouse model of stroke (53). The authors concluded that although there are still several limitations to overcome before the application of this technique in clinical practice, USPIO-enhanced MRI might provide useful surrogate markers for detecting a therapeutic effect in pre-clinical studies.

Another approach to the long-term monitoring of vascular system is represented by encapsulation of SPION into red blood cells in order to ensure their increased blood circulation time. As shown in a paper by Rahmer et al. (54), SPION-loaded RBCs can be imaged in the blood pool of mice several hours after injection, and their presence in circulation for up to $24 \mathrm{~h}$ was confirmed by spectroscopic quantification of the iron concentration in mouse blood samples collected after injection of SPION-loaded RBCs. Using this novel approach, long-term monitoring in cardiovascular diseases (e.g., monitoring the bleeding after stroke, imaging vessel architecture during interventional procedures, or controlling the treatment efficacy) can be envisioned without the necessity of the repeated administration of contrast agents.

\section{Nanosystems for vascular treatment and regeneration}

Although pharmacologic agents for the treatment of cardiovascular disorders are available, the conventional therapy using systemic delivery methods has several serious drawbacks, such as considerable side-effects or 
low efficacy at tolerated doses. To overcome the problems associated with traditional therapeutic approaches, the targeted nanoparticles can be used as transport vehicles that allow local targeted drug delivery to disease-specific cells or tissues and thus concentrate the therapeutic agent at the site of action. In this manner, drug cytotoxicity is expected to be reduced by (a) targeted tissue accumulation and (b) reduction of the required dosis. Additionally, as the nanocarrier systems are larger than $5 \mathrm{~nm}$ in diameter (ca. 10-200 nm) they evade renal clearance thus increasing circulation half-life of the transported drugs.

\section{Nano-sized drug carriers}

Among the materials most commonly used for cardiovascular drug-delivery systems are the nanoparticles or nanoshells made of natural or synthetic polymers, such as liposomes and lipidots, dextrans, poly(lactic-co-glycolic acid) (PLGA), polyaccrylates, as well as metal or metal oxide nanoparticles (e.g., gold, silver, SPION), and quantum dots. Several of the commonly tested drug-carrier systems [reviewed in detail in (55)] are briefly outlined below.

Liposomes are composed of a lipid bilayer consisting of amphipathic phospholipids (primarily phosphatidylcholine) that enclose an interior aqueous space (56). The head groups of phospholipids are usually functionalized with maleimide, which allows conjugation to antibodies or other ligands, and/or with polymerizable moieties to improve stability [e.g., polyethylene glycol (PEG)-ylated stealth liposomes]. Among the drug-delivery systems, liposomes have relatively low toxicity and a good therapeutic index $(56,57)$. A subgroup of those compounds, cationic liposomes, originally used as transfection reagents for gene or siRNA delivery, can be easily functionalized with antibodies or ligands. Among their advantages as a drugdelivery platform are the ease of preparation, commercial availability and overall low immunogenicity (58), which is expected to enable safe and repeated administration.

PLGA, poly(lactic-co-glycolic acid), is the most common biodegradable polymer Federal Drug Agency (FDA)-approved for use in humans. As PLGA degradation products (lactic acid and glycolic acid) are easily metabolized and easily eliminated from the body, the systemic toxicity associated with PLGA application is low (59).

Dextrans are stable glucose polymers that contain functional groups for derivatization (60). Apart from stability, several other advantages such as water solubility, and drug protection from degradation which allows sustained release of active compounds, make them a suitable platform for delivering pharmaceutical agents (61).
Gold nanoparticles consisting of a dielectric core of silica coated with a metallic layer of gold, are available in various sizes and forms (62) and can be used for e.g., as biosensors, or photoactive agents for optical imaging, for photothermal ablation therapy, or as drug carriers. SPIONs consist of iron oxide core, often coated with organic materials such as fatty acids, polysaccharides, or polymers $(63,64)$. The magnetic properties of SPIONs allow the remote control of their accumulation by means of external magnetic field, as well as their application for hyperthermia-therapy. Conjugation SPIONs with drugs, in combination with an external magnetic field to target the nanoparticles (so called "magnetic drug targeting", Figure 1), has additionally emerged as a promising strategy of drug delivery, which results in increased drug payloads in the target tissue, at the same time reducing their systemic dose and toxicity as demonstrated by the in vivo studies from our group (65-67).

\section{Cell-based therapies}

Due to the safety concerns associated with the use of cell-labeling strategies, development of low side-effect agents for tracking of the stem cells has been challenging (69). Because of this, it is difficult to evidence the fate of those cells in the human studies, despite the promising results with regard to their regenerative capacities in cardiovascular diseases. In one of the pioneering works, Himes et al. (70) used embryonic stem cells loaded with SPION for long-term monitoring of their fate following intramyocardial injection in a mouse model of MI. The subsequent studies by Sadek et al. (71) utilized the USPIObased contrast agent ferumoxide (Endorem/Feridex IV) in combination with transfection agent protamine sulfate for labeling and tracking of bone marrow-derived human mononuclear cells and $\mathrm{C} 2 \mathrm{C} 12$ skeletal myoblasts within rat myocardium. In the settings of MI, SPION-labeling of mesenchymal stem cells (MSCs) was furthermore utilized for cell tracking and the simultaneous evaluation of their long-term therapeutic potential i.e., left ventricular ejection fraction assessment (72). SPION-enhanced MRI has moreover been tested for tracking endothelial progenitor cells in a rat model of MI (73).

To demonstrate the potential of nano-labels for stem cell tracking during ischemic brain injury, ferumoxideprotamine sulfate label has been used for MSC labeling in the experimental cerebral infarction (74). Ferumoxide has also been tested as an MRI label for human neural stem cells (75). Other studies utilized microgel iron oxide nanoparticles (76), and SPION-loaded cationic 
A

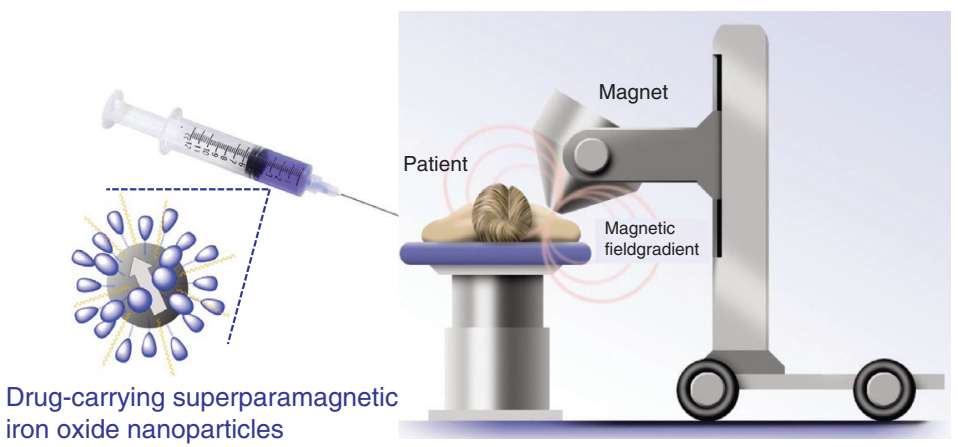

B
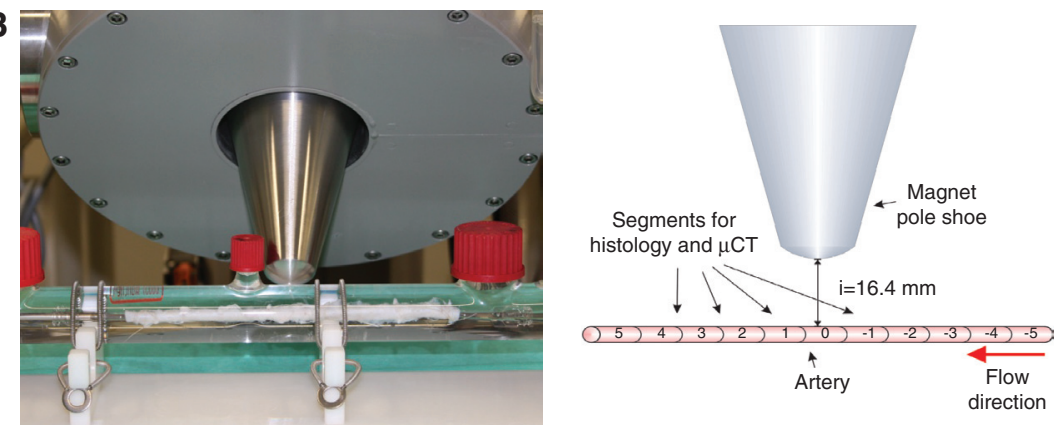

C
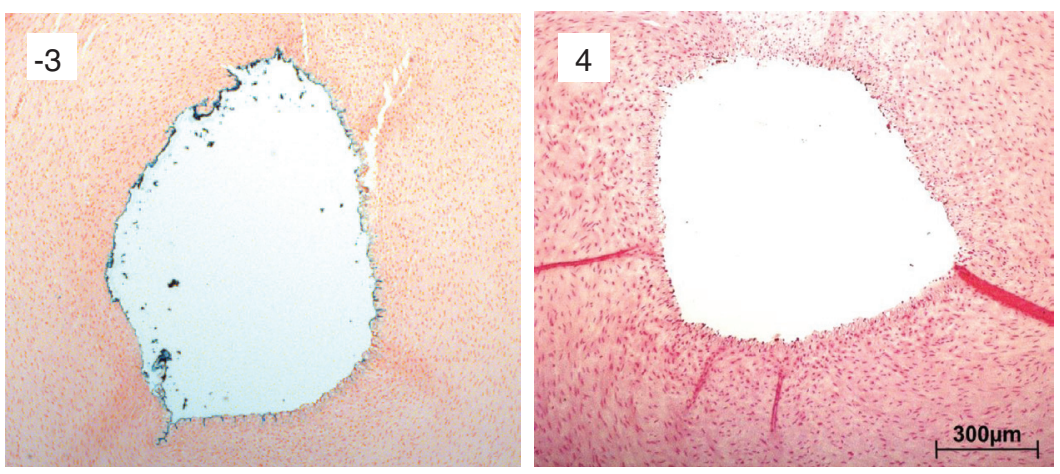

Figure 1 Magnetic drug targeting.

(A) Principle of the MDT method; (B) Superparamagnetic iron oxide nanoparticle accumulation in a bovine artery using magnetic field: Photo shows the ex vivo bovine artery model (left panel), experimental setup is schematically presented in the right panel; (C) Histochemical analysis of iron accumulation (Prussian blue staining) in the aortic segments relative to the magnet positioning (68).

nanovessicles (77) in different models of murine stroke for labeling human and rat MSCs, respectively. Wang et al. (78) developed a novel fluorescent-magnetitenanocluster with high MRI sensitivity and high labeling efficiency for MSCs, which allowed tracking their migration and accumulation in the ischemic region in a mouse stroke model. Similar results were obtained using fluorescent mesoporous silica-coated SPION for labeling neural progenitor cell (79). Both intravenous administration and implantation of such labeled cells in the mouse brain hemisphere contralateral to the ischemic injury region allowed tracking their migration to the lesion site using MRI. Taken together, these studies indicate that SPIONs represent a highly effective platform for labeling and MRI tracking of therapeutic stem cells in the context of CVD.

In a most recent study by Riegler et al. (80), MSC loading with SPION was used in order to magnetically target the cells to the sites of vascular injury induced by balloon angioplasty in rabbits. This study demonstrated the feasibility of magnetic cell delivery approach for localized accumulation of therapeutic cells in the injured arterial regions.

\section{Stroke treatment}

After cerebral ischemia and reperfusion, the oxidative stress and inflammation may contribute to the 
post-ischemic brain injury, affecting patients' outcomes. Nanoparticles can be used for targeted delivery of large payloads of antioxidant enzymes or reactive oxygen species scavengers into the affected tissue. This approach has been tested in mouse ischemic brain using platinum nanoparticles (Pt-NPs), which themselves are potent scavengers of superoxide anion $(81,82)$. Compared with vehicle, treatment with Pt-NPs significantly improved the motor function and greatly reduced the superoxide production and the infarct volume, indicating that the antioxidant properties of Pt-NPs can contribute to neuroprotection following the ischemic stroke $(81,82)$.

In their recent paper, Yun et al. (83) generated various nanoparticles (liposomes, polybutylcyanoacrylate (PBCA), or PLGA) that contained active superoxide dismutase (SOD), and were tagged with antibodies directed against the N-methyl-D-aspartate (NMDA) receptor 1 . In a mouse model of cerebral ischemia, the nanoparticles containing SOD showed protection against ischemia and reperfusion injury when applied after stroke with a 50\%$60 \%$ reduction in infarct volume, reduced inflammatory markers, and improved behavior in vivo.

Another treatment approach utilizing PEGylated-lipid nanoparticles that go across the blood-brain barrier was reported by Lu et al. (84). The nanoparticles encapsulating 3-n-butylphthalide were conjugated to Fas ligand antibody that selectively present on brain ischemic region. Those nanoparticles effectively accumulated in ischaemic region of mouse brain, and resulted in significant improvements in brain injury and in neurological deficit after ischaemia, with the significantly reduced dosages vs. free 3-n-butylphthalide. These studies show that targeted nanoparticles containing protective factors may be viable candidates for the treatment of stroke-induced ischemic brain injury.

\section{Thrombolysis}

Rapid recanalization of an occluded artery is essential for better outcomes in acute myocardial infarction or stroke. The current fibrinolytic therapy can be rapidly administered, but does not achieve a high reperfusion rate and is associated with considerable side-effects (85). Additionally, many patients are ineligible for systemic thrombolytic therapy, e.g., due to delayed admission to the hospital after symptom onset, or because of recent surgery, bleeding, etc. Development of delivery systems for rapid thrombolysis, characterized by a strong fibrinolytic effect and low bleeding risk, is therefore one of the most urgent tasks in cardiovascular medicine.
Thrombin represents the most important target of direct anticoagulants within the coagulation cascade. Two recent publications from the group of Wickline $(86,87)$ investigated the effects of the nanoparticle-bound potent thrombin inhibitor, d-phenylalanyl-1-prolyl-l-arginylchloromethyl ketone (PPACK) in a mouse model of acute arterial thrombosis due to the photochemical injury of the carotid artery. PPACK-perfluorocarbon nanoparticles outperformed both heparin and uncomplexed PPACK in inhibiting thrombosis, and formed a local clotting barrier that continued to manifest clot inhibition even as systemic effects rapidly diminished (86). Administration of PPACKliposomes prior to the injury significantly delayed the time to arterial occlusion as compared to free PPACK. Systemic anticoagulant profiles revealed a rapid return to control levels within $50 \mathrm{~min}$, whereas the antithrombin activity was maintained at the injury site (87). The establishment of a potent and long-acting anticoagulant surface over a newly forming clot with the use of thrombin targeted nanoparticles that do not require systemic anticoagulation to be effective offers an alternative site-targeted approach to the management of acute thrombosis.

Current thrombolytic therapy by infusion of tPA is characterized by several drawbacks, including low efficacy combined with a high risk of bleeding complications (85). Therefore, several innovative strategies aiming at targeted and/or local applications of plasminogen activators have been designed. The possibility of magnetic-targeting of tPA for local thrombolysis was investigated by Ma et al. in a rat embolic model (88). Polyacrylic acid-coated magnetite nanoparticles bound to TPA (tPA equivalent of 0.2 $\mathrm{mg} / \mathrm{kg}$ ) were administered intraarterially under guidance with the external magnet moving back and forth along the iliac artery. tPA-NPs restored the iliac blood flow within $75 \mathrm{~min}$ to $82 \%$ of that before the clot lodging, whereas equivalent amount of free tPA exerted no improvement on hemodynamics. The authors concluded that magnetic tPA-NPs allow reproducible and effective target thrombolysis with $<20 \%$ of the regular dose of free tPA.

Recently, a novel drug delivery nanosystem was described comprising tPA, basic gelatin and zinc acetate (89). Within this nanosystem, tPA activity was reduced in vitro to approximately $50 \%$ of free tPA and was fully recoverable by the application of low frequency ultrasound. In a swine acute myocardial infarction model, plasma tPA activity after intravenous injection of nanoparticles was approximately $25 \%$ of free tPA and was recovered completely by transthoracic ultrasound application, with significantly higher tPA activity near the affected coronary artery than in the femoral artery region. In comparison to treatment with free tPA $(0.447 \mathrm{mg} / \mathrm{kg})$, 
which recanalized the occluded coronary artery in only 1 of 10 swine, nanoparticles containing the same dose of tPA with ultrasound activation achieved recanalization in 9 of 10 swine within $30 \mathrm{~min}$, suggesting that this nanosystem bears promising potential for improved thrombolysis.

Another novel and extremely promising nanomedical strategy of targeted tPA delivery to stenotic arteries employing hemodynamic forces was recently described by Korin et al. (90). Since occlusions in blood vessels result in local increases in shear stress leading to platelet activation and clotting, the authors designed micro-aggregates of PLGA nanoparticles coated with tPA. These microaggregates are not affected by physiologic flow conditions with shear stress values up to $70 \mathrm{dyn} / \mathrm{cm}^{2}$, but exposed to abnormally high shear stress in the regions of vascular occlusion/stenosis, undergo break up followed by local drug release. As compared with free drug, the shear-activated tPA-coated nanoparticles induced rapid dissolution of arterial thrombi induced by the exposure of mouse mesenteric arteries to ferric chloride, with complete clearance of occluding thrombi within 5 min after application (90). Moreover, upon infusion of lethally large fibrin clots, the immediate application of the shear-activated tPA-coated nanoparticles increased survival by $80 \%$. The doses of shear-activated tPA-nanoparticles required for clot dissolution were about 100-times lower than the doses required for achieving comparable effects with free drug (90). This strategy, utilizing a universal hemodynamic phenomenon of shear stress increase upon reduction in vessel diameter should result in a broad applicability for all occlusive vascular conditions, including e.g., treatment of stenotic atherosclerotic plaques, pulmonary emboli, and ischemic stroke.

\section{Myocardial infarction}

Regeneration of the infarcted heart is one of the most important therapeutic goals in cardiovascular medicine. In search for a suitable nanotechnological platform for regenerative and anti-remodeling drug delivery, Paulis et al. (91) investigated the penetration of different paramagnetic lipid nanoconstructs (micelles, $15 \mathrm{~nm}$ in size, or liposomes, ca. $100 \mathrm{~nm}$ in size) in the infarct region. The authors showed that both in acute and chronic myocardial infarction settings, micelles permeate the entire infarct area, and can thus represent a good system for the delivery of cardioprotective drugs and for non-invasive monitoring of the infarct size by MRI (91). Several recent publications have furthermore tested whether nanoparticulate drug- or gene-delivery is capable of stimulating the regeneration of ischemically damaged hearts. Binsalamah et al. (92) utilized chitosanalginate nanoparticles loaded with proangiogenic and cardioprotective placental growth factor (PlGF). The intramyocardial injection of these constructs provided a sustained slow-release PIGF therapy, resulting in decreased scar formation, improved left ventriculat function and an anti-inflammatory systemic cytokine profile. Similar approach was tested by Chang et al. (93), who utilized PLGA nanoparticles conjugated with insulin-like growth factor-1 (IGF-1). Single post-MI intramyocardial injection of these nanoparticles resulted in prolonged retention of IGF-1 in the myocardium, which was sufficient to prevent cardiomyocyte apoptosis, and to reduce infarct size and improve left ventricular function at 21 days after MI.

Gene therapy represents yet another possibility to improve patients' outcomes following MI. Zhang et al. (94) applied externally controlled magnetic nanobeads conjugated to adenoviral vectors-encoded human vascular endothelial growth factor (VEGF) gene. Following intravenous administration, the nanobeads were accumulated in the myocardial region by external epicardial magnet, resulting in a good transduction efficiency and a strong VEGF gene expression in the ischemic zone of the heart. This led to improved left ventricular function, increased capillary and arteriolar density and reduced the collagen deposition in infarcted region, indicating that magnetic targeting enhances local transduction efficiency, and supports cardiac repair. Gene silencing was also tested in a recent study by Liu et al. (95) as a promising tool for regulating gene expression following MI. Oligo-arginineconjugated dendrimer loaded with siRNA against angiotensin 2 type 1 receptor (AT1R) prevented the receptor upregulation in vivo and improved the recovery of cardiac function after the ischemia-reperfusion myocardial injury. These studies demonstrate the enormous potential of nanoparticle-based technology for improved clinical therapy of MI and ischemic CVD.

\section{Plaque stabilization}

Atherosclerotic plaque stabilization is the aim of the current pharmacologic strategies, including statin therapy. As the experimental approaches to nanoparticlebased treatment of different stages of atherosclerosis are reviewed in detail elsewhere (10), this paragraph will only briefly list some of the potential targets for lesion stabilization. As an example, interventions with an inhibitory effect on macrophages have thus far been tested in ApoE-deficient mice utilizing pitavastatin-loaded PLGA nanoparticles (96). As compared with pitavastatin alone, 
the nanoparticle-mediated delivery of pitavastatin to circulating inflammatory monocytes prevented plaque destabilization and rupture by inhibiting their inflammatory activity and recruitment to the lesions. In a study by McCarthy et al., the administration of magnetofluorescent nanoparticles with light-activated therapeutic moieties which allow phototoxic activation (97), allowed an efficient focal ablation of inflammatory macrophages upon irradiation of the plaques. Such nanoparticles inducing focal toxicity confined to macrophages, without affecting endothelial or smooth muscle cells (SMCs), could have a durable plaque-stabilizing effect.

Another vital target for plaque-stabilizing therapies is represented by plaque neovascularization, as shown by the studies of Winter et al. (98) who applied $\alpha_{v} \beta_{3}$ integrintargeting SPION for site-specific delivery of antiangiogenic drug fumagillin in a rabbit model of atherosclerosis. These studies demonstrated superior antiangiogenic activity and reduced toxicity of $\alpha_{v} \beta_{3}$-targeting, fumagillin-carrying nanoparticles, as compared with systemic drug application (98). Targeted nanoparticles for local drug delivery can thus improve the therapeutic effect of current pharmacologic compounds for plaque stabilization.

\section{Application of nanoparticles to prevent in-stent restenosis}

Stent implantation allows recanalization of stenosed vessels, but is often related with complications, such as stent thrombosis and restenosis. Stent thrombosis is induced by the disruption of the endothelial monolayer and necessitates lengthy dual-antiplatelet therapy (DAPT). Stent implantation additionally results in an excessive SMC proliferation, which in the longer term can cause restenosis and vessel occlusion $(99,100)$. To prevent this process, drug-eluting stent (DES) containing anti-proliferative drugs are used. In clinical trials, DES has been shown to significantly reduce restenosis as compared to bare metal stents $(101,102)$. However, drug-induced inhibition of SMC proliferation also inhibits the re-establishment of a healthy endothelium, thus increasing the risk of stent-related thrombosis (103-105). Therefore, new stent systems targeting SMCs without adverse effects on endothelial cells are urgently needed. Several nanotechnological approaches to this issue have been reported, some of which are discussed below.

Polymer liposome nanoparticles targeted to chondroitin sulfate proteoglycans that encapsulated prednisolone were tested in a study by Joner et al. in order to prevent neointimal hyperplasia following bare metal stent implantation in rabbit (106). These nano-constructs specifically targeted the sites of stent-induced injury (106), increased the tissue concentration of prednisolone in stented arteries by 100 -fold as compared to contralateral nonstented arteries, and resulted in significant suppression of in-stent neointimal growth (106). This indicates that site-specific targeting of anti-inflammatory drug-loaded nanoparticles to the stented arteries can constitute a suitable method for the prevention of in-stent restenosis.

Another approach was tested by Tsukie et al. (107), who utilized a novel bioabsorbable polymeric nanoparticle-eluting stent (NES) that provides more sustained delivery of therapeutic agents than the common dip-coated DES (108). For this purpose, nanoparticles were produced containing pitavastatin. In a pig coronary artery stent model, the effectivity of in-stent stenosis inhibition by statin-NES equaled that of polymer-coated sirolimus-eluting stents, but was not accompanied by delayed endothelialization as observed in the sirolimus group. These findings indicate that inhibition of in-stent stenosis without delaying endothelial healing is possible (107).

A promising strategy to increase the rate of stent endothelialization was reported by Polyak et al. (109). In this study, endothelial cells expressing luciferase were preloaded with biodegradable polymeric superparamagnetic nanoparticles in order to enable their magnetic targeting to the steel surfaces of intraarterial stents. In the presence of a uniform external magnetic field, magnetic nanoparticle-loaded bovine aortic endothelial cells were successfully targeted to stents implanted in rat carotid arteries. Optical imaging confirmed significantly greater luciferase expression at the stented arteries treated with magnetically-labeled endothelial cells compared with nonmagnetic controls. Nanotechnology thus offers multiple strategies for improving the safety profiles of stents following cardiovascular interventions.

\section{Tissue engineering and vessel endothelialization}

Functionalized tissue-engineered vascular grafts do not only possess potential for applications in peripheral and coronary bypass surgery but are also attractive to the pediatric surgery for congenital heart defects. Cell loading with magnetic nanoparticles is one of nanotechnologic strategies that have been often applied to vascular tissue fabrication. This technique allows the deposition of loaded fibroblasts, SMCs and endothelial cells on the luminal side of the tubular scaffolds by means of external magnetic force as described by Ito, Perea and GonzalezMolina (110-113). Moreover, the magnetic tissue fabrication (114), was recently tested in vitro for cardiac tissue 
engineering. Using cardiomyocytes labeled with magnetic nanoparticles, the formation of a ring-shaped tissue that possessed a multilayered cell structure and contractile properties was achieved. These results indicate that magnetic tissue fabrication is a promising approach both for vessel and for cardiac tissue engineering.

\section{Clinical applications: state of the art}

Comparing with the vast number of bench research reports focusing on cardiovascular applications of nanotechnologies that have been published in the recent years, the reported clinical trials are scarce (Table 1). Below, several early cardiovascular imaging studies using magnetic nanoparticles and the few recent pilot trials involving nanosystems are highlighted.

\section{Pilot studies - cardiovascular imaging}

The majority of the clinical trials involving nanoparticles dates back to the beginning of the previous decade (20012004). These studies, most of which originated from the group of J.H. Gillard, utilized USPIO-based contrast agents in order to detect and characterize atherosclerotic plaques, based on the specific incorporation of USPIO by activated macrophages [reviewed in (136)].

Early clinical studies using ferumoxtran (Sinerem/ Combidex) have shown that USPIO accumulate in atherosclerotic plaques in aorta and pelvic arteries (115), as well as carotid plaques $(116,117)$, resulting in areas of focal signal loss on in vivo MR images that correspond to accumulation of iron particles in ex vivo specimens. A study by Kooi et al. (117) performed on 11 symptomatic patients scheduled for carotid endarterectomy demonstrated that USPIO accumulated predominantly in macrophages in ruptured and rupture-prone atherosclerotic lesions, whereas hardly any USPIO were taken up in stable plaques (117).

A clinical study from the group of J.H. Gillard confirmed the ability of USPIO-enhanced MRI to identify plaque inflammation by accumulation of USPIO within macrophages in stenotic carotid plaques (118). In that study, areas of signal intensity reduction, corresponding to USPIO- and macrophage-positive histological sections, were visualized in 7 of 8 patients receiving ferumoxtran. These data were subsequently validated on 30 symptomatic patients scheduled for carotid endarterectomy, showing USPIO enhancement in 90\% patients with severe stenosis (119). A more recent study used USPIO to compare 10 patients with symptomatic and 10 with asymptomatic carotid stenosis (137). In symptomatic patients, significantly more focal areas of signal drop were observed than in asymptomatic group, indicating increased inflammatory infiltrates. Interestingly, focal areas of signal reduction were also detected in some asymptomatic plaques suggesting that USPIO-enhanced MRI is capable of identifying inflammation within otherwise morphologically "stable" plaques. Subsequent USPIO studies, performed in 40 patients with carotid stenosis (120), confirmed that the patients with asymptomatic carotid atheroma contralateral to the symptomatic disease showed more inflammatory activity than the completely asymptomatic cohort, despite a mean lower grade of luminal stenosis (46\% vs. 63\%). These findings were corroborated by a further study that compared the degree of inflammation on USPIO-enhanced imaging between asymptomatic carotid plaques in patients with coronary artery disease (CAD) and in individuals with a carotid stenosis who were completely asymptomatic in all vascular regions (121). Patients with CAD had more inflammatory activity within their carotid atheroma than did the completely asymptomatic cohort despite a mean lower degree of luminal stenosis (59\% vs. 65\%). The authors concluded that inflammatory activity may be a significant risk factor in asymptomatic disease and USPIO-enhanced MRI may prove a useful technique to improve the risk stratification of patients with carotid stenosis. In 2013, a new study from the group of J.H. Gillard was published, investigating for the first time the feasibility of longitudinal sequential MR imaging before and $36 \mathrm{~h}$ after USPIO infusion at 0, 6, and 12 months, in 10 patients with a moderate asymptomatic carotid stenosis (122). The patients, none of whom received pharmacotherapy, remained asymptomatic within the course of the study and there was no statistical difference in their USPIO uptake between the three time points. Comparing the quadrant signal before USPIO infusion, a good agreement over the 1-year period was observed. The quadrant signal detected after USPIO infusion was in a good agreement between 0 and 6 months, and in moderate agreement between 0 and 12 months, suggesting that inflammation within the carotid plaque is a changeable and dynamic process. Apart from important information on quantitative reproducibility of the technique, this study provided evidence that within the 6 months, USPIO nanoparticles were cleared out of the atherosclerotic plaque. Importantly, no adverse effects following multiple USPIO infusions were observed (122), indicating that this technique is clinically safe and applicable, also for the future longitudinal studies involving pharmacologic interventions. 


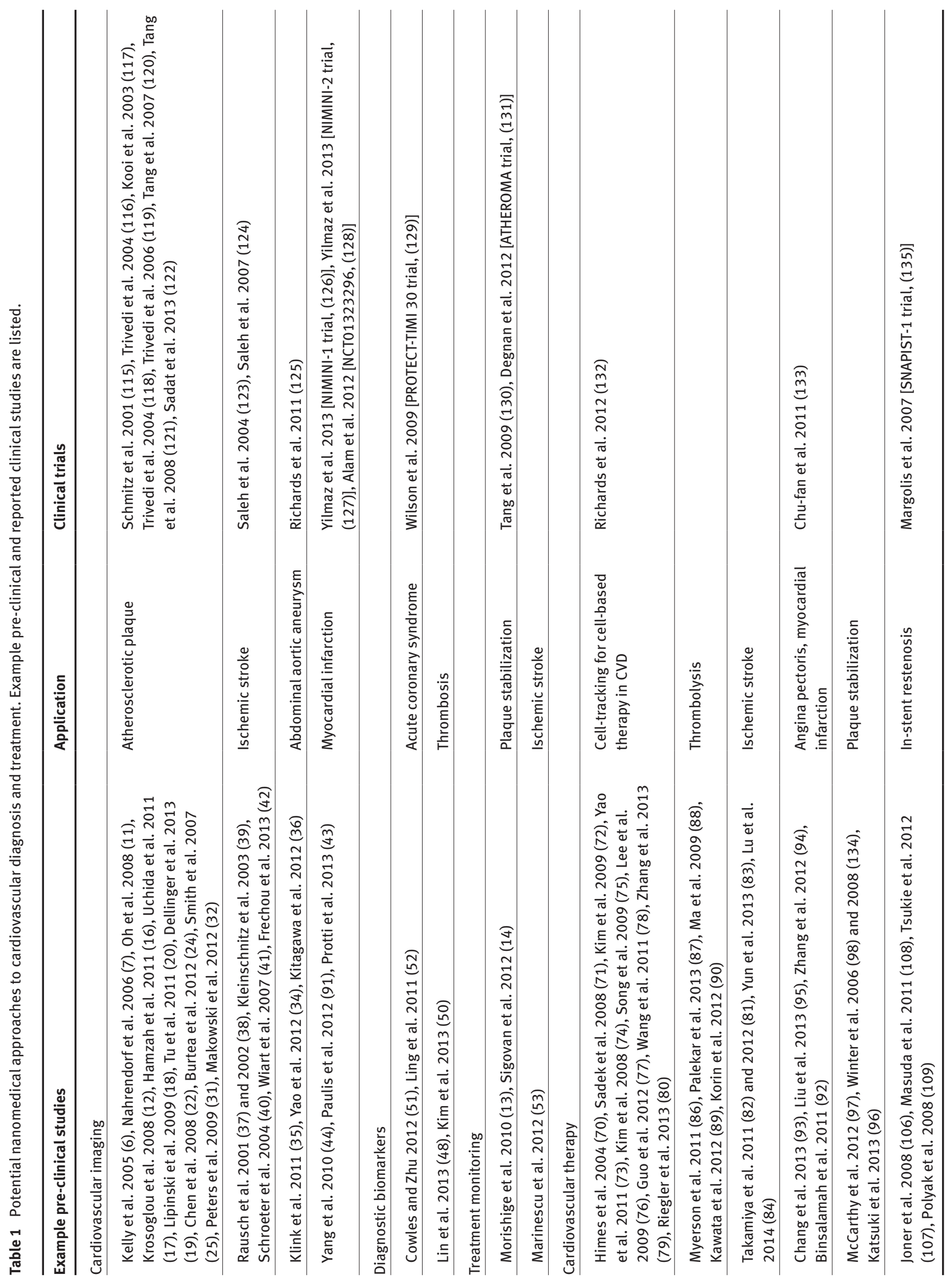


The feasibility of USPIO-enhanced MRI to detect inflammation following the ischaemic stroke was investigated by Saleh et al. (123). In this clinical phase II study, 10 consecutive patients received ferumoxtran (Sinerem/Combidex) infusion at the end of the first week after symptom onset. Two follow-up MRI scans were performed, at 24-36 $\mathrm{h}$ and 48-72 $\mathrm{h}$ after infusion. USPIO-induced signal alterations representing parenchymal enhancement were different from conventional gadolinium-enhanced MRI (Magnevist ${ }^{\circledR}$, Schering), showed an increase over time, and corresponded to the distribution of macrophages. The authors concluded that increasing USPIO-enhancement on T1-weighted images indicates brain infiltration by USPIO-laden macrophages, and may provide an in vivo surrogate marker of cellular inflammation in stroke. More recently, the utility of USPIO-enhanced MRI for estimating macrophage infiltration into early ischemic stroke lesions was examined in another study from the same group (124). Patients with stroke received intravenous ferumoxtran followed by four subsequent MRI scans. In 3 of 9 analyzed patients, parenchymal USPIO enhancement was observed on T1-weighted spin-echo images. USPIO-dependent signal changes reflected the variable extent and distribution of macrophage infiltration in different lesion types, indicating that USPIO-enhanced MRI may help tailoring the anti-inflammatory therapy in patients with stroke.

As a further possible application, USPIO-enhanced MRI was tested for prediction of expansion and rupture of life-threatening aortic aneurysms (125). As their prognosis currently relies on the measurement of aneurysm diameter only, new techniques are urgently needed to assess the rate of AAA expansion. The study was carried out in 29 stable patients with asymptomatic AAA, who received MRI scans before and 24-36 h after administration of ferumoxtran. The study demonstrated that the patients with distinct mural uptake of USPIO had a 3-fold higher AAA growth rate than those with no or nonspecific USPIO uptake despite having similar aneurysm diameters. This indicated that the uptake of USPIO in AAA is capable of identifying cellular inflammation and can distinguish between slow and rapidly progressive aneurysm expansion, thus improving risk stratification in the patients.

Most recently, Yilmaz et al. $(126,127)$, tested the suitability of USPIO-based MRI contrast agents for characterization of myocardial infarct pathology as compared with conventional gadolinium-based imaging. The studies showed that the approved dose of ferucarbotran (Resovist ${ }^{\circledR}$, NIMINI-1 trial) did not allow improved visualization of myocardial peri-infarct zone as compared to gadolinium-based contrast agent Magnevist ${ }^{\circledR}$ (126). In contrast, the administration of ferumoxytol (Rienso/
Feraheme $^{\mathrm{TM}}$, NIMINI-2 trial) in 14 patients with myocardial infarction allowed a better characterization of the injured myocardium and inflammatory macrophage accumulation, as well as the extent and composition of the periinfarct zone, as compared with Magnevist ${ }^{\circledR}$ (127). Nearly in parallel, another clinical study utilizing ferumoxytol for USPIO-enhanced MRI to assess cellular myocardial inflammation following acute myocardial infarction was published (128). In line with the data of Yilmaz et al., this study showed a strong USPIO accumulation in the infarct tissue of patients with recent myocardial infarction, and a less pronounced uptake in the peri-infarct and remote myocardium. These findings indicate that a new generation of USPIO formulations with a favorable safety profile (138) allows non-invasive detection and characterization of the infarcted myocardium and rise hope for the rapid development of nanoparticulate and easily functionalized contrast agents with superior clinical and prognostic value.

\section{Pilot studies -treatment monitoring and cardiovascular biomarkers}

Apart from providing prognostic information and aiding disease diagnosis, nanoparticles can constitute a useful tool for monitoring the treatment efficacy and act as biomarkers for therapeutic interventions. Below, several clinical feasibility studies are described that highlight these important applications.

USPIO-enhanced MRI was first used to monitor the effects of aggressive vs. mild lipid-lowering therapy on macrophage burden in carotid plaques, in a randomized controlled study performed by the group of J.H. Gillard [ATHEROMA study, (130)]. Patients with moderate carotid stenosis, who demonstrated intraplaque accumulation of USPIO (ferumoxtran) on MRI at the baseline, received either $10 \mathrm{mg}$ or $80 \mathrm{mg}$ atorvastatin daily for 12 weeks. Twenty patients completed the treatment in each group. A significant reduction from baseline in USPIO-defined inflammation was observed in the 80-mg group at both 6 weeks and at 12 weeks. Moreover, aggressive lipid-lowering therapy for 12 weeks was associated with significant reduction in USPIO-defined inflammation as compared to the mild lipid-lowering treatment (130). This technique was thus suitable to assess therapeutic response in an interventional drug trial in humans, additionally facilitating enrollment of the specific patient cohort in the trial. The results of the long-term follow-up of the ATHEROMA trial were published in 2012 (131), evaluating the ability of USPIOenhanced MRI to predict subsequent cerebrovascular 
and cardiovascular events. In those analyses, 62 patients initially screened for enrollment to ATHEROMA trial were examined for the occurrence of adverse cerebrovascular or cardiovascular events following the initial USPIO-imaging. Despite the small size of the study group and only 17 cardiovascular/cerebrovascular events reported in total, an association was observed ( $\mathrm{p}=0.07$ ) between the magnitude of maximal USPIO-induced signal intensity loss within carotid plaques and the risk of developing subsequent vascular events (131). As the study lacked adequate statistical power, future prospective studies with new generation of USPIO-based contrast agents are urgently needed. Such studies should incorporate long-term follow-up analyses in order to estimate the usefulness of USPIO-enhanced MRI for the assessment of future event risk in asymptomatic patients with carotid atherosclerosis.

Cell-based therapies are another attractive option for treatment of cardiovascular diseases. In this context, a safe and reliable method of tracking the cells in vivo to ensure the delivery of sufficient cell numbers to the diseased region is critical for the therapy development (69, 139). In their recent paper, Richards et al. (132) report the development of GMP-compliant method of labeling peripheral blood mononuclear cells (PBMCs) with SPION, and their successful tracking by MRI in humans. Labeling of the mononuclear cells with ferumoxides (Endorem/ Feridex IV) did not affect their viability, migration or cytokine release in vitro, and allowed their MRI identification in vivo for at least 7 days. A phased-dosing study, demonstrated that systemic delivery of up to $10^{9}$ SPIONlabeled cells in humans is safe and does not affect any hematological, biochemical, or coagulation variables. Therefore, 12 healthy volunteers received $10^{8}-10^{9}$ SPIONlabeled cells approximately $27 \mathrm{~h}$ after a local cutaneous inflammation was induced in the thigh by intradermal injection of tuberculin. Intravenously delivered SPIONlabeled cells were tracked to the inflamed skin at 24 and $48 \mathrm{~h}$ post-administration, as visualized by MRI and confirmed using Prussian blue staining of inflamed skin biopsies. The authors concluded that SPION-labeling is a safe and feasible technique that has a major potential for cardiovascular applications including monitoring of cell therapies and tracking inflammatory cells by MRI.

Myocardial injury estimated by cardiac troponin plasma or serum levels often goes unnoticed due to the low sensitivity of the current generation assays. In 2009, a pilot study was performed to evaluate the clinical value of a new ultra-sensitive nanoparticle assay for cardiac troponin I (nano-cTnI; detection limit $0.0002 \mu \mathrm{g} / \mathrm{L}$ ) based on the sandwich antibody technique with chemical signal enhancement of gold nanoparticles to which the secondary antibody was bound [PROTECT-TIMI 30 trial, (129)]. In this study, blood samples from two cohorts were re-analysed: 50 patients with unstable angina and serial negative cTnI using current generation troponin assay, and 50 patients with definite myocardial infarction who had an initially negative current generation cTnI result, but results of sampling at 6-8 and 18-24 h revealed increase in cTnI. In the first cohort classified as UAP, $44 \%, 62 \%$, and $82 \%$ of patients had an elevated nano-cTnI result measured at 0 , 2 , and $8 \mathrm{~h}$ with the nano-cTnI assay. In patients with definite myocardial injury but an initially negative cTnI, $72 \%$ and $98 \%$ had a positive nano-cTnI score $(>0.003 \mu \mathrm{g} / \mathrm{L})$ at 0 and $2 \mathrm{~h}$. Thus, using a nanoparticle assay for cTnI, myocardial injury was detectable in a substantial proportion of patients previously classified as having unstable angina pectoris. The emergence of a new generation of troponin assays has the potential to improve the diagnosis of myocardial infarction based on an enhanced analytical performance at very low concentrations of troponin.

\section{Pilot studies - disease treatment}

In comparison to diagnostic applications, nanomedical approaches to treatment of cardiovascular disorders in humans remain limited. Two published pilot trials investigating the feasibility of nanoparticle-based drug delivery systems for treatment of angina pectoris and in-stent restenosis are briefly described below.

Angina pectoris resulting from myocardial ischemia affects about $50 \%$ of all patients with CAD. In a study by Chu-fan et al. (133), prostaglandin E1 (PGE1), an endogenous vasodilatory mediator effective in the treatment of critical limb ischemia, was tested in patients with angina pectoris undergoing a percutaneous coronary intervention (PCI). A randomized controlled trial utilizing PGE1 incorporated into lipid microspheres (lipo-PGE1) was conducted in 79 patients. Intravenous administration of lipoPGE1 $(20 \mu \mathrm{g} /$ day for 5 days, starting at least $48 \mathrm{~h}$ before PCI) was well tolerated, with no serious adverse events or side-effects. With regard to the therapeutic effect, cardiac troponin $\mathrm{T}$ and creatine kinase myocardial isoenzyme concentrations were lower in the lipo-PGE1 group than in the control group at $6 \mathrm{~h}, 12 \mathrm{~h}$ and $24 \mathrm{~h}$ after PCI. The incidence of postprocedural myocardial injury was reduced in the lipo-PGE1 group by ca. 20\% compared with the control group, indicating that lipo-PGE1 may improve patients' outcomes following the elective PCI.

In-stent restenosis remains a significant limitation to the long-term patency of vascular stents. Drug-eluting stents inhibit restenosis but are associated with increased 
risk of stent thrombosis (103). The only published safety and dose-finding human trial utilizing nanoparticle-based drug delivery system for in-stent restenosis (SNAPIST-I trial) utilized a novel 130-nm, albumin-bound particle form of paclitaxel (nab-paclitaxel) (135). Patients with angina received nab-paclitaxel at $10,30,70$, or 100 $\mathrm{mg} / \mathrm{m}^{2}$ intravenously after bare metal stenting of de novo lesion in a single coronary artery. Data obtained for all 23 enrolled patients indicated that no significant adverse events were attributable to the nab-paclitaxel at 10 or $30 \mathrm{mg} / \mathrm{m}^{2}$, although moderate neutropenia, sensory neuropathy and mild to moderate reversible alopecia occurred at higher doses. At 2 months post-procedure, no major adverse cardiac events were reported, whereas 4 target lesions required revascularization for restenoses at 6 months. The authors concluded that intravenous application of nab-paclitaxel was well tolerated at doses below $70 \mathrm{mg} / \mathrm{m}^{2}$, suggesting that systemic nab-paclitaxel may be used with any available bare-metal stent and at potentially lower cost than DES (135). To date however, no evaluation is available as to the clinical efficacy of nab-paclitaxel for the suppression of coronary in stent restenosis.

\section{Obstacles and considerations}

\section{Imaging}

In spite of the promising results of the pilot studies in humans, the marketing and the clinical application of iron oxide-containing contrast agents are at the stillstand. Although SPIO and USPIO have been approved for clinical use in the past, currently they are scarcely available (see Table 2), with the exception of the oral iron oxide contrast agent, ferumoxsil (Lumirem/Gastromark), and ferumoxytol (Rienso/Feraheme), a novel intravenous agent approved for iron replacement therapy in chronic renal failure patients with iron-deficiency anemia.

The nephrotoxicity related to gadolinium-based contrast agents remains a concern. Because of the nanoparticle accumulation occurring typically in liver, spleen, kidneys and bladder, the retention of gadolinium can lead to a delayed serious adverse reaction known as nephrogenic systemic fibrosis in patients with impaired renal function (140). Although SPION/USPIO had been reported to have favorable safety profiles (141), the delayed toxicity effects due to an increased inflammation and oxidative stress cannot be excluded (142). New generation of iron oxide-based contrast agents with superior safety profile and targeting properties is thus needed for the future clinical imaging of atherosclerotic plaques, myocardial infarction, and ischemic stroke (138).

\section{Drug-delivery systems}

Nanoparticle-based drug delivery systems are an attractive platform to improve the efficacy and reduce the systemic toxicity of cardiovascular drugs. For the purpose of therapeutic applications, novel nanoparticle formulations, including drug-carrying liposomes, lipidots, and polyaccrylates are currently being developed. In order to deliver the therapeutic nanoparticles locally and to monitor the treatment efficacy, additional modifications are required, such as grafting of targeting-moiety and/or image contrast enhancement, leading to the development of complex multifunctional nanoparticles (Figure 2). However, manufacturing such intelligent, tissue- or cell-specific "theranostic" nanoparticles necessitates additional production

Table 2 SPIO- and USPIO-based contrast agents tested in pre-clinical and clinical trials. Source: http://www.mr-tip.com/.

\begin{tabular}{|c|c|c|c|c|}
\hline Product & Trade name (EU) & Trade name (USA) & Availability & Marketed by (EU/USA) \\
\hline Ferumoxides & Endorem & Feridex IV & Discontinued in 2008 & Guerbet/Berlex BayerHealthcare \\
\hline Ferrixan (Ferucarbotran) & Resovist (Cliavist) & & $\begin{array}{l}\text { Approved in EU 2001, } \\
\text { production abandoned in } 2009\end{array}$ & Bayer Shering Pharma AG \\
\hline Ferumoxtran-10 & Sinerem & Combidex & $\begin{array}{l}\text { Marketing authorization } \\
\text { withdrawn in } 2007\end{array}$ & Guerbet/AMAG Pharma \\
\hline Feruglose & Clariscan & $\begin{array}{l}\text { NC100150, PEG- } \\
\text { feron }\end{array}$ & $\begin{array}{l}\text { Development discontinued due } \\
\text { to safety concerns }\end{array}$ & GE Healthcare \\
\hline Ferumoxil & Lumirem & Gastromark & $\begin{array}{l}\text { FDA-approved 1996, available } \\
\text { for sale }\end{array}$ & Guerbet/AMAG Pharma \\
\hline Ferumoxytol & Rienso & Feraheme & $\begin{array}{l}\text { FDA approved in } 2009 \text { for } \\
\text { iron-replacement therapy; EU } \\
\text { marketing authorization in } \\
2012\end{array}$ & Takeda/AMAG Pharma \\
\hline
\end{tabular}


steps and increases the costs of synthesis. Additionally, much more detailed characterization methods, as well as complex behavior in vivo must be considered, which increase the regulatory hurdles and hinder the clinical translation (143). Some of these considerations are discussed in detail below.

\section{Nanoparticle characterization}

Compared to the free drugs, nanosystems are extremely complex constructs and the lack of their comprehensive standardized characterization is a serious obstacle to overcome before they can obtain the approval for use in humans. Whereas high-sensitivity diagnostic nanosystems for ex vivo determination of the urine or serum biomarkers are likely to enter clinical practice in the near future, in vivo drug-delivery systems must be subject to a close toxicologic and pharmacologic scrutiny prior to the application in patients.

Nanoparticle chemical composition is often the most the critical feature that affects their toxicity. For example silver nanoparticles are reportedly more cytotoxic than gold nanoparticles $(144,145)$, but also dextran administration has been associated with some side effects, such as anaphylaxis, volume overload, pulmonary and cerebral oedema, or platelet dysfunction $(146,147)$. Acute renal failure is another rarely occurring but serious complication of dextran osmotic effect (148). Therefore, in patients with history of renal insufficiency, diabetes mellitus, or vascular disorders, large volumes of dextran-based drugs should be administered with particular caution.

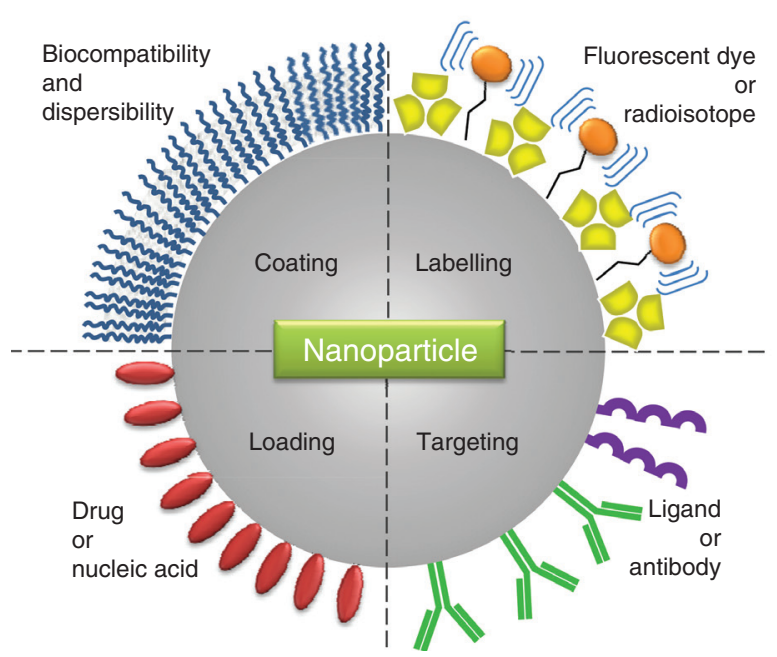

Particle surface charge, indicated by zeta potential, has a strong influence on their stability in biologic fluids. Nanoparticles with a zeta potential above $( \pm) 30 \mathrm{mV}$ are usually stable in suspension, as the surface charge prevents their aggregation. Surface charge additionally influences the biologic effects: in vivo studies have shown that cationic liposomes can cause dose-dependent toxicity and pulmonary inflammation (149). Size is another of the critical factors that affect the behaviour of nanoparticles (150). An inherent difficulty related to its standardized characterization is that nanoparticle size can dramatically vary between different dispersion media, depending on their ionic strength and protein content. Generally, 10-100 nm particle size is considered optimal for drug delivery; the smaller size can cause undesirable effects such as passing through the blood-brain barrier, and the particles with diameter $<5 \mathrm{~nm}$ are renally cleared which dramatically reduces their circulation time. In contrast, for delivery of drugs or imaging agents to atherosclerotic plaques, the nanoparticle diameter should not exceed 100 $\mathrm{nm}$, as larger nanoparticles do not penetrate the vessel wall readily. Due to the small sizes of nanoparticles, their surface area is large. This provides various possibilities of surface modifications in order to stabilize and prevent aggregation of nanoparticles, and to allow conjugation of ligands or drugs. However, functionalization of the nanoparticles with ligands or antibodies can additionally contribute to their immunogenicity. Moreover, stability of surface coatings within biological environment and the possible side effects of degradation products must be considered. Nanoparticle agglomeration, which occurs due to their large surface area-to-volume ratio, is a factor that may affect their toxicity (151). Agglomeration is influenced by

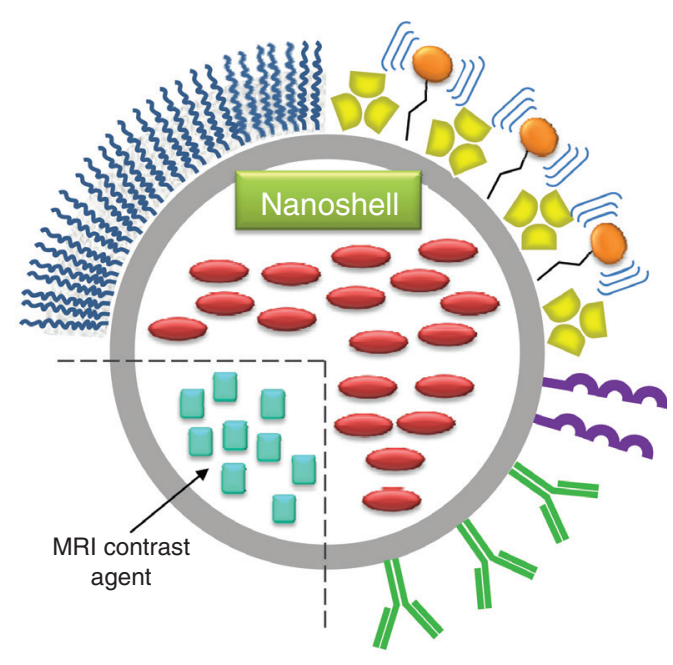

Figure 2 Possible modifications of nanoparticles. 
the particle composition, size and zeta potential, but also extrinsic factors, e.g., temperature, as well as $\mathrm{pH}$, osmotic strength and the presence of serum in the dispersion media. For clinical applications, nanoparticle agglomeration may be a key factor limiting their use in patients, as it affects the physicochemical properties, bioavailability, and thus efficacy. As aggregated nanoparticles are no longer nano-sized, they undergo a rapid recognition by reticuloendothelial system (RES) and are cleared by the liver or spleen. Moreover, their presence in circulation may cause serious undesirable side-effects, such as clogging blood or lymphatic vessels (152). Prevention of agglomeration is therefore required for designing a stable, clinically safe nanosystem. In this respect, PEGylation of nanoparticles is considered a good solution to reduce their agglomeration and toxicity, and to increase their circulation time. By creating a hydrophilic layer around the nanoparticles, PEGylation provides a strong steric barrier to opsonin adsorption (153), therefore preventing nanoparticle recognition by the RES and increasing their circulation half-life.

The detailed and standardized characterization of the above-mentioned physico-chemical properties in physiologic fluids is the key issue to consider before any given nanosystem can enter the preclinical in vitro and in vivo testing. As different techniques for nanoparticle characterization are available (e.g., transmission electron microscopy, Fourier transform infrared spectroscopy, dynamic light scattering), each of them featuring its own advantages and limitations, optimally the characterization data obtained with several different measurement methods should be routinely compared to ensure reliable results. Detailed characterization can facilitate the prediction of nanoparticle effectivity and toxicity in physiologic conditions and contribute to an improved design of stable, non-immunogenic constructs.

\section{Nanoparticle toxicity}

Characterization of the biological responses elicited by the nanosystems is critical for their future clinical applications. Although the concept of nanomedicine encompasses a localized delivery of nanosystems to target organs or tissues, their extended circulation time, as well as multiple degradation products, may result in cyto- and genotoxicity (154), as well as immunogenicity (155). Thus far, however, the basic cause-effect relationships are either not clearly demonstrated, or remain largely unexplored (156). Hence, detailed studies are urgently needed to identify various nanoparticle characteristics that can predict their toxicity. Due to this fact, nanotoxicology has emerged as an important area in the field of nanomedicine (152).
As stated above, physicochemical properties that may contribute to toxic effects of nanomaterials include chemical composition, charge, agglomeration state, particle size and surface properties (55). Among the currently investigated nanomaterials, the best safety profile i.e., the lowest toxicity was reported for PLGA, which thus has the best potential to be used in clinical applications. In the case of liposomes, the features that can predict their systemic toxicity are mainly related to their lipid composition and charge. Those characteristics should thus be considered when designing liposome-based drug-delivery systems in order to minimize the potential side effects $(149,157)$. Silica and titanium dioxide nanoparticles were reported to cross placenta barrier in mice and cause neurotoxicity in fetus, but these effects are abolished upon surface modification with carboxyl and amine groups (158). Among the metal oxide nanoparticles, iron oxide nanoparticles were reported to be non-cytotoxic for endothelial cells in vitro at concentrations below $0.1 \mathrm{mg} / \mathrm{mL}$ (159). Nonetheless, upon intravenous administration, their accumulation in liver and/or target tissue may potentially cause iron overload. Increased amounts of free iron can affect iron homeostasis and induce oxidative stress, leading to DNA damage and/or inflammation (142). However, the recent clinical study by Sadat et al. (122) provided evidence that USPIO nanoparticles are cleared out of the atherosclerotic plaques within 6 months post-application, and no adverse effects following multiple USPIO infusions were observed, confirming that these particles are clinically safe and well tolerated (138).

Other considerations related to nanosystem toxicity include their pyrogenicity and endotoxin contamination. Depending on the chemical/biological components and the production process, the final product may contain bacterial endotoxins (160), which can cause inflammatory response upon in vivo application, leading to the organ damage. The FDA-recommended high-sensitivity bacterial endotoxin test (limulus amoebocyte lysate assay) is commonly used in preclinical pharmaceutical development, but most nanoparticles interfere with the assay $(160,161)$. Alternate nanoparticle-compliant pyrogenicity tests are therefore urgently needed to assure the sterility of nanoparticles produced for clinical use (160).

In general, the toxicity of any nanosystem should first be evaluated in vitro on cultured cells, under conditions that resemble or mimic the physiological state in order to produce relabile data that predict in vivo responses (162). Following these extensive initial tests to assess the effect of nanoparticles on the components of blood, the firstcontact cells (e.g., endothelial cells in the case of intravenous application) and the target cells, the regulatory toxicity studies in animals, usually rats and mice, should 
be performed. Due to their size, nanoparticles typically remain in the circulation for considerable periods of time, and their in vivo behaviour and interactions with cellular and extracellular substrates may induce undesired effects, including hemolytic reactions, platelet and complement activation, reactive oxygen species production and genotoxicity. To assess acute single/repeated dose toxic effects and estimate maximum tolerated dose of nanosystems in vivo, animals should be monitored over 14 days for body weight, organ weight indices, as well as behavioural, biochemical and histopathological changes. Longer time span is necessary to evaluate nanoparticlemediated immunotoxicity and chronic toxicity. Moreover, the determination of pharmacokinetics, biodistribution, as well as the clearance rate and routes of degradation products must be performed (163), which is usually done by the detection of radiolabeled constituents in animal tissues harvested at different time points. In case of complex multi-component nanosystems, the expense and the efforts required for these investigations are immense.

Characterization of nanoparticle-based agents in an animal system is an essential part of assessing both nanotoxicology and in vivo efficacy, and a vital requirement to fulfill before any nanosystem is approved for clinical use by the regulatory bodies. However, the number of difficulties associated with these safety assessment studies is enormous, as analyses of all individual constituents of a complex multicomponent nanoparticle are required. Nanotoxicologic characterization is thus an important area that must undergo both extensive development and prompt standardization to ensure nanosystems approval for use in humans $(156,163)$.

\section{Scale-up and GMP production}

Another major hurdle to overcome in the process of nanosystem approval for clinical use is the GMP-compliant production (164). Additionally, the research and development methods often involve a low volume production and, in case of some manufacturing technologies, scaling up the process may pose serious difficulties. Apart from this, the costs of synthesis must be considered, as well as the batch-to-batch reproducibility (143). The latter issue is a serious problem, and may lead to devastating clinical consequences as recently reported for ferumoxytol (Rienso), the whole batch of which had to be withdrawn from the Swiss market due to one case of death and several cases of drug-hypersensitivity. Therefore, the extensive batch characterization and safety assessments are required, as well as the chemical engineering and pharmaceutical efforts, to provide a platform for a large-scale production of highquality therapeutic nanosystems.

\section{Administration route}

Lack of effective delivery methods constitutes one of the major obstacles hindering the development and clinical application of therapeutic nanosystems (165). In terms of patients' acceptance, the best delivery route is usually represented by oral administration, however, few vasculoprotective nanoparticle formulations are thus far available in this form [e.g., PLGA-curcumin $(166,167)$, heparinloaded nanoparticles $(168,169)]$. Parenteral administration evades the problems related to gastrointestinal route, i.e., the poor absorption, gastrointestinal intolerance and/ or non-compliance associated with oral preparations. However, parenteral administration of nanodrugs commonly tested in animal studies is suboptimal, as it necessitates a careful patient monitoring and can be expected to have a very low acceptance in patients. Joint efforts of bioengineers, toxicologists, clinicians and pharmacologists will be necessary to design and synthesize targeted drug-delivery systems which will combine an improved pharmacokinetics and biodistribution of the therapeutics with the patient-accepted formulation.

\section{Regulatory issues}

During the approval process of any novel drugs, the candidates are closely scrutinized with regard to their safety and efficacy in humans. Standard drugs are usually composed of a single active agent combined with inactive formulation aids. As the nanodrugs often represent multicomponent formulations, new considerations arise concerning the regulated drug development and testing (143, 163). Thus far, due to the lack of clear guidelines and regulations applicable to nanomedicines, the responsible regulatory agencies examine each nanoparticle-based drug on a product-by-product basis. The in vivo behaviour of the multi-component nanoconstructs can, however, differ from the activities enticed by single-component system. Precise new guidelines for nanoparticle characterization are necessary, as well as uniform standardized methods for testing the toxicity and efficacy of nanosystems in preclinical animal models, in order to provide a transparent regulatory frame for the pharmaceutical industry.

Taken together, whereas the implementation of novel nanoparticle-based ex vivo diagnostic assays for cardiovascular biomarkers analysis can only be limited by the complexity of production or the high costs, much more 


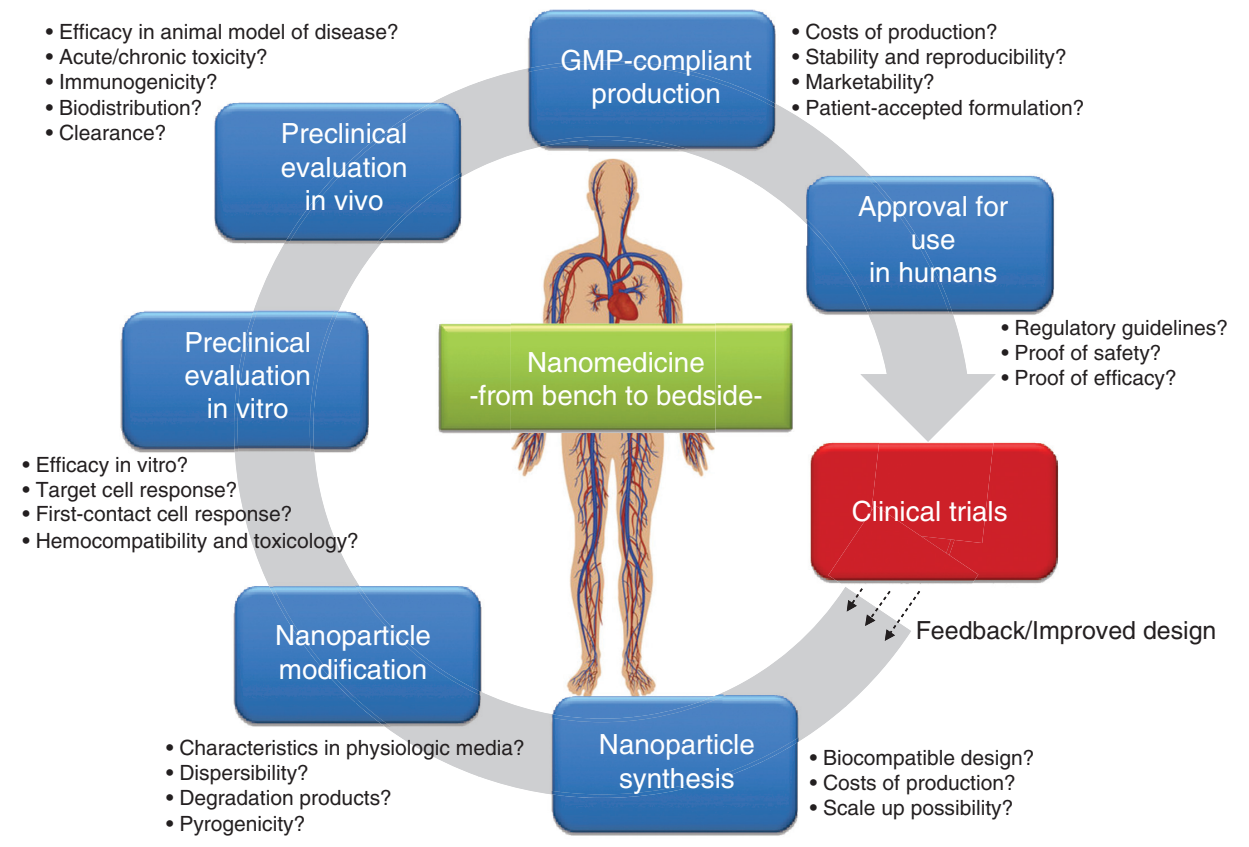

Figure 3 The bench-to-bedside translation steps for nanoparticle-based imaging and drug-delivery systems. Important considerations related to each step are highlighted.

serious obstacles hinder the clinical application of nanoparticles for cardiovascular imaging or drug delivery (Figure 3). As shown by the promising results of the pilot clinical studies, imaging of atherosclerosis using USPIOenhanced MRI harbours tremendous potential to monitor the disease progression and to influence prognosis, both in asymptomatic population, as well as in subjects at risk of future or recurrent cardiovascular events. Imaging nanosystems composed of e.g., approved nanoparticle-based contrast agents and a targeting moiety have a good chance to pass the in vivo efficacy and toxicity tests favourably and obtain the approval for clinical use. However, even then substantial clinical research will be necessary to verify the clinical utility and identify the category of patients in whom this type of evaluation will translate into the biggest clinical benefits. Although routine bedside application of nanoparticle-based cardiovascular drug-delivery systems still belongs to a distant future, interdisciplinary efforts and collaborative research programs move cardiovascular nanomedicine a step closer to reality.

\section{From bench to bedside: conclusions}

Despite multiple technical and regulatory constraints that currently hinder the widespread clinical application, the potential clinical impact of nanotechnology in terms of managing strategies to ultimately reduce the occurrence of cardiovascular events is enormous. The translation of basic research studies into the clinical trials represents one of the biggest challenges in this field. Apart from safety and efficacy issues, legal and financial aspects must be considered to ensure a rapid and secure implementation of the new techniques in clinical practice. Moreover, the acceptance of the industry is required in order to establish GMP-compliant production and marketing. Extensive efforts in the field of cardiovascular nanomedicine together with promising results of animal studies and early clinical trials rise hope that, before long, safe nanosystems integrating a theranostic component and a targeting moiety will be approved for the diagnosis and therapy of cardiovascular diseases. As the clinical experience remains limited, careful evaluation of nanosystems' safety, as well as the validation of their diagnostic and prognostic value in clinical trials are urgently needed.

Acknowledgments: This work was supported by the EU project FP7-NMP-2012-LARGE-6-309820, the DFG grant CI162/2-1, and the Emerging Field Initiative of the University of Erlangen-Nuremberg project "TOP-Biomat”.

Received January 8, 2014; accepted March 5, 2014; previously published online April 9, 2014 


\section{References}

1. Lozano R, Naghavi M, Foreman K, Lim S, Shibuya K, Aboyans V, et al. Global and regional mortality from 235 causes of death for 20 age groups in 1990 and 2010: a systematic analysis for the Global Burden of Disease Study 2010. Lancet 2012;380: 2095-128.

2. Lim SS, Vos T, Flaxman AD, Danaei G, Shibuya K, Adair-Rohani $\mathrm{H}$, et al. A comparative risk assessment of burden of disease and injury attributable to 67 risk factors and risk factor clusters in 21 regions, 1990-2010: a systematic analysis for the Global Burden of Disease Study 2010. Lancet 2012;380:2224-60.

3. Hahn MA, Singh AK, Sharma P, Brown SC, Moudgil BM. Nanoparticles as contrast agents for in-vivo bioimaging: current status and future perspectives. Anal Bioanal Chem 2011;399:3-27.

4. Nahrendorf M, Zhang H, Hembrador S, Panizzi P, Sosnovik DE, Aikawa E, et al. Nanoparticle PET-CT imaging of macrophages in inflammatory atherosclerosis. Circulation 2008;117:379-87.

5. Wang T, Mancuso JJ, Sapozhnikova V, Dwelle J, Ma LL, Willsey $B$, et al. Dual-wavelength multifrequency photothermal wave imaging combined with optical coherence tomography for macrophage and lipid detection in atherosclerotic plaques using gold nanoparticles. J Biomed Opt 2012;17:036009.

6. Kelly KA, Allport JR, Tsourkas A, Shinde-Patil VR, Josephson L, Weissleder R. Detection of vascular adhesion molecule-1 expression using a novel multimodal nanoparticle. Circ Res 2005;96:327-36.

7. Nahrendorf M, Jaffer FA, Kelly KA, Sosnovik DE, Aikawa E, Libby $\mathrm{P}$, et al. Noninvasive vascular cell adhesion molecule-1 imaging identifies inflammatory activation of cells in atherosclerosis. Circulation 2006;114:1504-11.

8. Reynolds PR, Larkman DJ, Haskard DO, Hajnal JV, Kennea NL, George AJ, et al. Detection of vascular expression of E-selectin in vivo with MR imaging. Radiology 2006;241:469-76.

9. Jacobin-Valat MJ, Deramchia K, Mornet S, Hagemeyer CE, Bonetto S, Robert R, et al. MRI of inducible P-selectin expression in human activated platelets involved in the early stages of atherosclerosis. NMR Biomed 2011;24:413-24.

10. Cicha IL, Alexiou C, Garlichs CD. Nanomedicine in diagnostics and therapy of cardiovascular diseases: beyond atherosclerotic plaque imaging. Nanotechnol Rev 2013;2:449-72.

11. Oh J, Feldman MD, Kim J, Sanghi P, Do D, Mancuso JJ, et al. Detection of macrophages in atherosclerotic tissue using magnetic nanoparticles and differential phase optical coherence tomography. J Biomed Opt 2008;13:054006.

12. Korosoglou G, Weiss RG, Kedziorek DA, Walczak P, Gilson WD, Schar M, et al. Noninvasive detection of macrophage-rich atherosclerotic plaque in hyperlipidemic rabbits using "positive contrast" magnetic resonance imaging. J Am Coll Cardiol 2008;52:483-91.

13. Morishige K, Kacher DF, Libby P, Josephson L, Ganz P, Weissleder R, et al. High-resolution magnetic resonance imaging enhanced with superparamagnetic nanoparticles measures macrophage burden in atherosclerosis. Circulation 2010;122:1707-15.

14. Sigovan M, Kaye E, Lancelot E, Corot C, Provost N, Majd Z, et al. Anti-inflammatory drug evaluation in ApoE-/- mice by ultrasmall superparamagnetic iron oxide-enhanced magnetic resonance imaging. Invest Radiol 2012;47:546-52.
15. te Boekhorst BC, Bovens SM, Nederhoff MG, van de Kolk KW, Cramer MJ, van Oosterhout MF, et al. Negative MR contrast caused by USPIO uptake in lymph nodes may lead to false positive observations with in vivo visualization of murine atherosclerotic plaque. Atherosclerosis 2010;210:122-9.

16. Hamzah J, Kotamraju VR, Seo JW, Agemy L, Fogal V, Mahakian $L M$, et al. Specific penetration and accumulation of a homing peptide within atherosclerotic plaques of apolipoprotein E-deficient mice. Proc Natl Acad Sci USA 2011;108:7154-9.

17. Uchida M, Kosuge H, Terashima M, Willits DA, Liepold LO, Young MJ, et al. Protein cage nanoparticles bearing the LyP-1 peptide for enhanced imaging of macrophage-rich vascular lesions. ACS Nano 2011;5:2493-502.

18. Lipinski MJ, Frias JC, Amirbekian V, Briley-Saebo KC, Mani V, Samber D, et al. Macrophage-specific lipid-based nanoparticles improve cardiac magnetic resonance detection and characterization of human atherosclerosis. JACC Cardiovasc Imaging 2009;2:637-47.

19. Dellinger A, Olson J, Link K, Vance S, Sandros MG, Yang J, et al. Functionalization of gadolinium metallofullerenes for detecting atherosclerotic plaque lesions by cardiovascular magnetic resonance. J Cardiovasc Magn Reson 2013;15:7.

20. Tu C, Ng TS, Sohi HK, Palko HA, House A, Jacobs RE, et al. Receptor-targeted iron oxide nanoparticles for molecular MR imaging of inflamed atherosclerotic plaques. Biomaterials 2011;32:7209-16.

21. Frias JC, Williams KJ, Fisher EA, Fayad ZA. Recombinant HDL-like nanoparticles: a specific contrast agent for MRI of atherosclerotic plaques. J Am Chem Soc 2004;126:16316-7.

22. Chen W, Vucic E, Leupold E, Mulder WJ, Cormode DP, Briley-Saebo KC, et al. Incorporation of an apoE-derived lipopeptide in high-density lipoprotein MRI contrast agents for enhanced imaging of macrophages in atherosclerosis. Contrast Media Mol Imaging 2008;3:233-42.

23. Wen S, Liu DF, Liu Z, Harris S, Yao YY, Ding Q, et al. OxLDLtargeted iron oxide nanoparticles for in vivo MRI detection of perivascular carotid collar induced atherosclerotic lesions in ApoE-deficient mice. J Lipid Res 2012;53:829-38.

24. Burtea C, Ballet S, Laurent S, Rousseaux O, Dencausse A, Gonzalez W, et al. Development of a magnetic resonance imaging protocol for the characterization of atherosclerotic plaque by using vascular cell adhesion molecule-1 and apoptosis-targeted ultrasmall superparamagnetic iron oxide derivatives. Arterioscler Thromb Vasc Biol 2012;32:e36-48.

25. Smith BR, Heverhagen J, Knopp M, Schmalbrock P, Shapiro J, Shiomi $M$, et al. Localization to atherosclerotic plaque and biodistribution of biochemically derivatized superparamagnetic iron oxide nanoparticles (SPIONs) contrast particles for magnetic resonance imaging (MRI). Biomed Microdevices 2007;9:719-27.

26. van Tilborg GA, Vucic E, Strijkers GJ, Cormode DP, Mani V, Skajaa T, et al. Annexin A5-functionalized bimodal nanoparticles for MRI and fluorescence imaging of atherosclerotic plaques. Bioconjug Chem 2010;21:1794-803.

27. Winter PM, Morawski AM, Caruthers SD, Fuhrhop RW, Zhang $\mathrm{H}$, Williams TA, et al. Molecular imaging of angiogenesis in early-stage atherosclerosis with alpha(v)beta3-integrin-targeted nanoparticles. Circulation 2003;108:2270-4. 
28. Somanath PR, Malinin NL, Byzova TV. Cooperation between integrin alphavbeta3 and VEGFR2 in angiogenesis. Angiogenesis 2009;12:177-85.

29. Winter PM, Caruthers SD, Allen JS, Cai K, Williams TA, Lanza GM, et al. Molecular imaging of angiogenic therapy in peripheral vascular disease with alphanubeta3-integrin-targeted nanoparticles. Magn Reson Med 2010;64:369-76.

30. Flacke S, Fischer S, Scott MJ, Fuhrhop RJ, Allen JS, McLean M, et al. Novel MRI contrast agent for molecular imaging of fibrin: implications for detecting vulnerable plaques. Circulation 2001;104:1280-5.

31. Peters D, Kastantin M, Kotamraju VR, Karmali PP, Gujraty $\mathrm{K}$, Tirrell $\mathrm{M}$, et al. Targeting atherosclerosis by using modular, multifunctional micelles. Proc Natl Acad Sci USA 2009;106:9815-9.

32. Makowski MR, Forbes SC, Blume U, Warley A, Jansen CH, Schuster $A$, et al. In vivo assessment of intraplaque and endothelial fibrin in ApoE(-/-) mice by molecular MRI. Atherosclerosis 2012;222:43-9.

33. Thompson RW, Geraghty PJ, Lee JK. Abdominal aortic aneurysms: basic mechanisms and clinical implications. Curr Probl Surg 2002;39:110-230.

34. Yao Y, Wang Y, Zhang Y, Li Y, Sheng Z, Wen S, et al. In vivo imaging of macrophages during the early-stages of abdominal aortic aneurysm using high resolution MRI in ApoE mice. PLoS One 2012;7:e33523.

35. Klink A, Heynens J, Herranz B, Lobatto ME, Arias T, Sanders $\mathrm{HM}$, et al. In vivo characterization of a new abdominal aortic aneurysm mouse model with conventional and molecular magnetic resonance imaging. J Am Coll Cardiol 2011;58: 2522-30.

36. Kitagawa T, Kosuge H, Uchida M, Dua MM, lida Y, Dalman RL, et al. RGD-conjugated human ferritin nanoparticles for imaging vascular inflammation and angiogenesis in experimental carotid and aortic disease. Mol Imaging Biol 2012;14:315-24.

37. Rausch M, Sauter A, Frohlich J, Neubacher U, Radu EW, Rudin M. Dynamic patterns of USPIO enhancement can be observed in macrophages after ischemic brain damage. Magn Reson Med 2001;46:1018-22.

38. Rausch M, Baumann D, Neubacher U, Rudin M. In-vivo visualization of phagocytotic cells in rat brains after transient ischemia by USPIO. NMR Biomed 2002;15:278-83.

39. Kleinschnitz C, Bendszus M, Frank M, Solymosi L, Toyka $\mathrm{KV}$, Stoll G. In vivo monitoring of macrophage infiltration in experimental ischemic brain lesions by magnetic resonance imaging. J Cereb Blood Flow Metab 2003;23:1356-61.

40. Schroeter M, Saleh A, Wiedermann D, Hoehn M, Jander S. Histochemical detection of ultrasmall superparamagnetic iron oxide (USPIO) contrast medium uptake in experimental brain ischemia. Magn Reson Med 2004;52:403-6.

41. Wiart M, Davoust N, Pialat JB, Desestret V, Moucharrafie S, Cho $\mathrm{TH}$, et al. MRI monitoring of neuroinflammation in mouse focal ischemia. Stroke 2007;38:131-7.

42. Frechou M, Beray-Berthat V, Raynaud JS, Meriaux S, Gombert F, Lancelot $E$, et al. Detection of vascular cell adhesion molecule-1 expression with USPIO-enhanced molecular MRI in a mouse model of cerebral ischemia. Contrast Media Mol Imaging 2013;8:157-64.

43. Protti A, Dong X, Andia ME, Yu B, Dokukina K, Chaubey S, et al. Assessment of inflammation with a very small iron-oxide particle in a murine model of reperfused myocardial infarction. J Magn Reson Imaging 2014;39:598-608.

44. Yang Y, Yang Y, Yanasak N, Schumacher A, Hu TC. Temporal and noninvasive monitoring of inflammatory-cell infiltration to myocardial infarction sites using micrometer-sized iron oxide particles. Magn Reson Med 2010;63:33-40.

45. Jackson SP. Arterial thrombosis - insidious, unpredictable and deadly. Nat Med 2011;17:1423-36.

46. Dimarsico L, Cymet T. Pulmonary embolism - a state of the clot review. Compr Ther 2007;33:184-91.

47. Davie EW, Kulman JD. An overview of the structure and function of thrombin. Semin Thromb Hemost 2006;32(Suppl 1):3-15.

48. Lin KY, Kwong GA, Warren AD, Wood DK, Bhatia SN. Nanoparticles that sense thrombin activity as synthetic urinary biomarkers of thrombosis. ACS Nano 2013;7:9001-9.

49. Lenain N, Freund M, Leon C, Cazenave JP, Gachet C. Inhibition of localized thrombosis in P2Y1-deficient mice and rodents treated with MRS2179, a P2Y1 receptor antagonist. J Thromb Haemost 2003;1:1144-9.

50. Kim DE, Kim JY, Sun IC, Schellingerhout D, Lee SK, Ahn CH, et al. Hyperacute direct thrombus imaging using computed tomography and gold nanoparticles. Ann Neurol 2013;73:617-25.

51. Cowles CL, Zhu X. Dual signal amplification for bioassays using ion release from nanolabels and ion-activated enzyme kinetics. Analyst 2012;137:4815-21.

52. Ling Y, Pong T, Vassiliou CC, Huang PL, Cima MJ. Implantable magnetic relaxation sensors measure cumulative exposure to cardiac biomarkers. Nat Biotechnol 2011;29:273-7.

53. Marinescu M, Chauveau F, Durand A, Riou A, Cho TH, Dencausse $A$, et al. Monitoring therapeutic effects in experimental stroke by serial USPIO-enhanced MRI. Eur Radiol 2013;23:37-47.

54. Rahmer J, Antonelli A, Sfara C, Tiemann B, Gleich B, Magnani $M$, et al. Nanoparticle encapsulation in red blood cells enables blood-pool magnetic particle imaging hours after injection. Phys Med Biol 2013;58:3965-77.

55. Sharma A, Madhunapantula SV, Robertson GP. Toxicological considerations when creating nanoparticle-based drugs and drug delivery systems. Expert Opin Drug Metab Toxicol 2012;8:47-69.

56. Puri A, Loomis K, Smith B, Lee JH, Yavlovich A, Heldman E, et al. Lipid-based nanoparticles as pharmaceutical drug carriers: from concepts to clinic. Crit Rev Ther Drug Carrier Syst 2009;26:523-80.

57. Balazs DA, Godbey W. Liposomes for use in gene delivery. J Drug Deliv 2011;2011:326-497.

58. Huwyler J, Drewe J, Krahenbuhl S. Tumor targeting using liposomal antineoplastic drugs. Int J Nanomedicine 2008;3:21-9.

59. Danhier F, Ansorena E, Silva JM, Coco R, Le Breton A, Preat V. PLGA-based nanoparticles: an overview of biomedical applications. J Control Release 2012;161:505-22.

60. Sun G, Mao JJ. Engineering dextran-based scaffolds for drug delivery and tissue repair. Nanomedicine (Lond) 2012;7: 1771-84.

61. Varshosaz J. Dextran conjugates in drug delivery. Expert Opin Drug Deliv 2012;9:509-23.

62. Menon JU, Jadeja P, Tambe P, Vu K, Yuan B, Nguyen KT. Nanomaterials for photo-based diagnostic and therapeutic applications. Theranostics 2013;3:152-66.

63. Wahajuddin, Arora S. Superparamagnetic iron oxide nanoparticles: magnetic nanoplatforms as drug carriers. Int J Nanomedicine 2012;7:3445-71. 
64. Tassa C, Shaw SY, Weissleder R. Dextran-coated iron oxide nanoparticles: a versatile platform for targeted molecular imaging, molecular diagnostics, and therapy. Acc Chem Res 2011;44:842-52.

65. Janko C, Durr S, Munoz LE, Lyer S, Chaurio R, Tietze R, et al. Magnetic drug targeting reduces the chemotherapeutic burden on circulating leukocytes. Int J Mol Sci 2013;14:7341-55.

66. Lyer S, Tietze R, Jurgons R, Struffert T, Engelhorn T, Schreiber $E$, et al. Visualisation of tumour regression after local chemotherapy with magnetic nanoparticles - a pilot study. Anticancer Res 2010;30:1553-7.

67. Tietze R, Lyer S, Durr S, Struffert T, Engelhorn T, Schwarz M, et al. Efficient drug-delivery using magnetic nanoparticles biodistribution and therapeutic effects in tumour bearing rabbits. Nanomedicine 2013;9:961-71.

68. Tietze R, Rahn H, Lyer S, Schreiber E, Mann J, Odenbach S, et al. Visualization of superparamagnetic nanoparticles in vascular tissue using XmuCT and histology. Histochem Cell Biol 2011;135:153-8.

69. Wang YX, Wang HH, Au DW, Zou BS, Teng LS. Pitfalls in employing superparamagnetic iron oxide particles for stem cell labelling and in vivo MRI tracking. Br J Radiol 2008;81:987-8.

70. Himes N, Min JY, Lee R, Brown C, Shea J, Huang X, et al. In vivo MRI of embryonic stem cells in a mouse model of myocardial infarction. Magn Reson Med 2004;52:1214-9.

71. Sadek H, Latif S, Collins R, Garry MG, Garry DJ. Use of ferumoxides for stem cell labeling. Regen Med 2008;3:807-16.

72. Kim YJ, Huh YM, Choe KO, Choi BW, Choi EJ, Jang Y, et al. In vivo magnetic resonance imaging of injected mesenchymal stem cells in rat myocardial infarction; simultaneous cell tracking and left ventricular function measurement. Int J Cardiovasc Imaging 2009;25(Suppl 1):99-109.

73. Yao Y, Li Y, Ma G, Liu N, Ju S, Jin J, et al. In vivo magnetic resonance imaging of injected endothelial progenitor cells after myocardial infarction in rats. Mol Imaging Biol 2011;13:303-13.

74. Kim D, Chun BG, Kim YK, Lee YH, Park CS, Jeon I, et al. In vivo tracking of human mesenchymal stem cells in experimental stroke. Cell Transplant 2008;16:1007-12.

75. Song M, Kim Y, Kim Y, Ryu S, Song I, Kim SU, et al. MRI tracking of intravenously transplanted human neural stem cells in rat focal ischemia model. Neurosci Res 2009;64:235-9.

76. Lee ES, Chan J, Shuter B, Tan LG, Chong MS, Ramachandra DL, et al. Microgel iron oxide nanoparticles for tracking human fetal mesenchymal stem cells through magnetic resonance imaging. Stem Cells 2009;27:1921-31.

77. Guo RM, Cao N, Zhang F, Wang YR, Wen XH, Shen J, et al. Controllable labelling of stem cells with a novel superparamagnetic iron oxide-loaded cationic nanovesicle for MR imaging. Eur Radiol 2012;22:2328-37.

78. Wang Y, Xu F, Zhang C, Lei D, Tang Y, Xu H, et al. High MR sensitive fluorescent magnetite nanocluster for stem cell tracking in ischemic mouse brain. Nanomedicine 2011;7: 1009-19.

79. Zhang L, Wang Y, Tang Y, Jiao Z, Xie C, Zhang H, et al. High MRI performance fluorescent mesoporous silica-coated magnetic nanoparticles for tracking neural progenitor cells in an ischemic mouse model. Nanoscale 2013;5:4506-16.

80. Riegler J, Liew A, Hynes SO, Ortega D, O’Brien T, Day RM, et al. Superparamagnetic iron oxide nanoparticle targeting of MSCs in vascular injury. Biomaterials 2013;34:1987-94.
81. Takamiya M, Miyamoto Y, Yamashita T, Deguchi K, Ohta Y, Abe K. Strong neuroprotection with a novel platinum nanoparticle against ischemic stroke- and tissue plasminogen activatorrelated brain damages in mice. Neuroscience 2012;221:47-55.

82. Takamiya M, Miyamoto Y, Yamashita T, Deguchi K, Ohta Y, Ikeda $Y$, et al. Neurological and pathological improvements of cerebral infarction in mice with platinum nanoparticles. J Neurosci Res 2011;89:1125-33.

83. Yun X, Maximov VD, Yu J, Zhu H, Vertegel AA, Kindy MS. Nanoparticles for targeted delivery of antioxidant enzymes to the brain after cerebral ischemia and reperfusion injury. J Cereb Blood Flow Metab 2013;33:583-92.

84. Lu YM, Huang JY, Wang H, Lou XF, Liao MH, Hong LJ, et al. Targeted therapy of brain ischaemia using Fas ligand antibody conjugated PEG-lipid nanoparticles. Biomaterials 2014;35:530-7.

85. Miller DJ, Simpson JR, Silver B. Safety of thrombolysis in acute ischemic stroke: a review of complications, risk factors, and newer technologies. Neurohospitalist 2011;1:138-47.

86. Myerson J, He L, Lanza G, Tollefsen D, Wickline S. Thrombininhibiting perfluorocarbon nanoparticles provide a novel strategy for the treatment and magnetic resonance imaging of acute thrombosis. J Thromb Haemost 2011;9:1292-300.

87. Palekar RU, Myerson JW, Schlesinger PH, Sadler JE, Pan $\mathrm{H}$, Wickline SA. Thrombin-targeted liposomes establish a sustained localized anticlotting barrier against acute thrombosis. Mol Pharm 2013;10:4168-75.

88. Ma YH, Wu SY, Wu T, Chang YJ, Hua MY, Chen JP. Magnetically targeted thrombolysis with recombinant tissue plasminogen activator bound to polyacrylic acid-coated nanoparticles. Biomaterials 2009;30:3343-51.

89. Kawata H, Uesugi Y, Soeda T, Takemoto Y, Sung JH, Umaki K, et al. A new drug delivery system for intravenous coronary thrombolysis with thrombus targeting and stealth activity recoverable by ultrasound. J Am Coll Cardiol 2012;60:2550-7.

90. Korin N, Kanapathipillai M, Matthews BD, Crescente M, Brill A, Mammoto T, et al. Shear-activated nanotherapeutics for drug targeting to obstructed blood vessels. Science 2012;337: 738-42.

91. Paulis LE, Geelen T, Kuhlmann MT, Coolen BF, Schafers M, Nicolay K, et al. Distribution of lipid-based nanoparticles to infarcted myocardium with potential application for MRI-monitored drug delivery. J Control Release 2012;162: 276-85.

92. Binsalamah ZM, Paul A, Khan AA, Prakash S, Shum-Tim D. Intramyocardial sustained delivery of placental growth factor using nanoparticles as a vehicle for delivery in the rat infarct model. Int J Nanomedicine 2011;6:2667-78.

93. Chang MY, Yang YJ, Chang CH, Tang AC, Liao WY, Cheng FY, et al. Functionalized nanoparticles provide early cardioprotection after acute myocardial infarction. J Control Release 2013;170:287-94.

94. Zhang Y, Li W, Ou L, Wang W, Delyagina E, Lux C, et al. Targeted delivery of human VEGF gene via complexes of magnetic nanoparticle-adenoviral vectors enhanced cardiac regeneration. PLoS One 2012;7:e39490.

95. Liu J, Gu C, Cabigas EB, Pendergrass KD, Brown ME, Luo Y, et al. Functionalized dendrimer-based delivery of angiotensin type 1 receptor siRNA for preserving cardiac function following infarction. Biomaterials 2013;34:3729-36. 
96. Katsuki S, Matoba T, Nakashiro S, Sato K, Koga JI, Nakano K, et al. Nanoparticle-mediated delivery of pitavastatin inhibits atherosclerotic plaque destabilization/rupture in mice by regulating the recruitment of inflammatory monocytes. Circulation 2014;129:896-906.

97. McCarthy JR, Korngold E, Weissleder R, Jaffer FA. A lightactivated theranostic nanoagent for targeted macrophage ablation in inflammatory atherosclerosis. Small 2010;6:2041-9.

98. Winter PM, Neubauer AM, Caruthers SD, Harris TD, Robertson JD, Williams TA, et al. Endothelial alpha(v) beta3 integrin-targeted fumagillin nanoparticles inhibit angiogenesis in atherosclerosis. Arterioscler Thromb Vasc Biol 2006;26:2103-9.

99. Farb A, Sangiorgi G, Carter AJ, Walley VM, Edwards WD, Schwartz RS, et al. Pathology of acute and chronic coronary stenting in humans. Circulation 1999;99:44-52.

100. Schwartz RS, Chronos NA, Virmani R. Preclinical restenosis models and drug-eluting stents: still important, still much to learn. J Am Coll Cardiol 2004;44:1373-85.

101. Moses JW, Leon MB, Popma JJ, Fitzgerald PJ, Holmes DR, O'Shaughnessy C, et al. Sirolimus-eluting stents versus standard stents in patients with stenosis in a native coronary artery. N Engl J Med 2003;349:1315-23.

102. Stone GW, Ellis SG, Cannon L, Mann JT, Greenberg JD, Spriggs $D$, et al. Comparison of a polymer-based paclitaxel-eluting stent with a bare metal stent in patients with complex coronary artery disease: a randomized controlled trial. J Am Med Assoc 2005;294:1215-23.

103. Joner M, Finn AV, Farb A, Mont EK, Kolodgie FD, Ladich E, et al. Pathology of drug-eluting stents in humans: delayed healing and late thrombotic risk. J Am Coll Cardiol 2006;48:193-202.

104. Iakovou I, Schmidt T, Bonizzoni E, Ge L, Sangiorgi GM, Stankovic $\mathrm{G}$, et al. Incidence, predictors, and outcome of thrombosis after successful implantation of drug-eluting stents. J Am Med Assoc 2005;293:2126-30.

105. Spertus JA, Kettelkamp R, Vance C, Decker C, Jones PG, Rumsfeld JS, et al. Prevalence, predictors, and outcomes of premature discontinuation of thienopyridine therapy after drug-eluting stent placement: results from the PREMIER registry. Circulation 2006;113:2803-9.

106. Joner M, Morimoto K, Kasukawa H, Steigerwald K, Merl S, Nakazawa G, et al. Site-specific targeting of nanoparticle prednisolone reduces in-stent restenosis in a rabbit model of established atheroma. Arterioscler Thromb Vasc Biol 2008;28:1960-6.

107. Tsukie N, Nakano K, Matoba T, Masuda S, Iwata E, Miyagawa $M$, et al. Pitavastatin-incorporated nanoparticle-eluting stents attenuate in-stent stenosis without delayed endothelial healing effects in a porcine coronary artery model. J Atheroscler Thromb 2013;20:32-45.

108. Masuda S, Nakano K, Funakoshi K, Zhao G, Meng W, Kimura $\mathrm{S}$, et al. Imatinib mesylate-incorporated nanoparticle-eluting stent attenuates in-stent neointimal formation in porcine coronary arteries. J Atheroscler Thromb 2011;18:1043-53.

109. Polyak B, Fishbein I, Chorny M, Alferiev I, Williams D, Yellen B, et al. High field gradient targeting of magnetic nanoparticleloaded endothelial cells to the surfaces of steel stents. Proc Natl Acad Sci USA 2008;105:698-703.
110. Perea H, Aigner J, Heverhagen JT, Hopfner U, Wintermantel E. Vascular tissue engineering with magnetic nanoparticles: seeing deeper. J Tissue Eng Regen Med 2007;1:318-21.

111. Perea H, Aigner J, Hopfner U, Wintermantel E. Direct magnetic tubular cell seeding: a novel approach for vascular tissue engineering. Cells Tissues Organs 2006;183:156-65.

112. Ito A, Ino K, Hayashida M, Kobayashi T, Matsunuma H, Kagami $\mathrm{H}$, et al. Novel methodology for fabrication of tissue-engineered tubular constructs using magnetite nanoparticles and magnetic force. Tissue Eng 2005;11:1553-61.

113. Gonzalez-Molina J, Riegler J, Southern P, Ortega D, Frangos CC, Angelopoulos Y, et al. Rapid magnetic cell delivery for large tubular bioengineered constructs. J R Soc Interface 2012;9:3008-16.

114. Akiyama H, Ito A, Sato M, Kawabe Y, Kamihira M. Construction of cardiac tissue rings using a magnetic tissue fabrication technique. Int J Mol Sci 2010;11:2910-20.

115. Schmitz SA, Taupitz M, Wagner S, Wolf KJ, Beyersdorff D, Hamm B. Magnetic resonance imaging of atherosclerotic plaques using superparamagnetic iron oxide particles. J Magn Reson Imaging 2001;14:355-61.

116. Trivedi RA, U-King-Im JM, Graves MJ, Kirkpatrick PJ, Gillard JH. Noninvasive imaging of carotid plaque inflammation. Neurology 2004;63:187-8.

117. Kooi ME, Cappendijk VC, Cleutjens KB, Kessels AG, Kitslaar PJ, Borgers $\mathrm{M}$, et al. Accumulation of ultrasmall superparamagnetic particles of iron oxide in human atherosclerotic plaques can be detected by in vivo magnetic resonance imaging. Circulation 2003;107:2453-8.

118. Trivedi RA, U-King-Im JM, Graves MJ, Cross JJ, Horsley J, Goddard MJ, et al. In vivo detection of macrophages in human carotid atheroma: temporal dependence of ultrasmall superparamagnetic particles of iron oxide-enhanced MRI. Stroke 2004;35:1631-5.

119. Trivedi RA, Mallawarachi C, U-King-Im JM, Graves MJ, Horsley J, Goddard MJ, et al. Identifying inflamed carotid plaques using in vivo USPIO-enhanced MR imaging to label plaque macrophages. Arterioscler Thromb Vasc Biol 2006;26:1601-6.

120. Tang TY, Howarth SP, Miller SR, Graves MJ, U-King-Im JM, Trivedi RA, et al. Comparison of the inflammatory burden of truly asymptomatic carotid atheroma with atherosclerotic plaques contralateral to symptomatic carotid stenosis: an ultra small superparamagnetic iron oxide enhanced magnetic resonance study. J Neurol Neurosurg Psychiatry 2007;78:1337-43.

121. Tang TY, Howarth SP, Miller SR, Graves MJ, U-King-Im JM, Li ZY, et al. Comparison of the inflammatory burden of truly asymptomatic carotid atheroma with atherosclerotic plaques in patients with asymptomatic carotid stenosis undergoing coronary artery bypass grafting: an ultrasmall superparamagnetic iron oxide enhanced magnetic resonance study. Eur J Vasc Endovasc Surg 2008;35:392-8.

122. Sadat U, Howarth SP, Usman A, Tang TY, Graves MJ, Gillard JH. Sequential imaging of asymptomatic carotid atheroma using ultrasmall superparamagnetic iron oxide-enhanced magnetic resonance imaging: a feasibility study. J Stroke Cerebrovasc Dis 2013;22:e271-6.

123. Saleh A, Schroeter M, Jonkmanns C, Hartung HP, Modder U, Jander S. In vivo MRI of brain inflammation in human ischaemic stroke. Brain 2004;127(Pt 7):1670-7. 
124. Saleh A, Schroeter M, Ringelstein A, Hartung HP, Siebler M, Modder $\mathrm{U}$, et al. Iron oxide particle-enhanced MRI suggests variability of brain inflammation at early stages after ischemic stroke. Stroke 2007;38:2733-7.

125. Richards JM, Semple SI, MacGillivray TJ, Gray C, Langrish JP, Williams $M$, et al. Abdominal aortic aneurysm growth predicted by uptake of ultrasmall superparamagnetic particles of iron oxide: a pilot study. Circ Cardiovasc Imaging 2011;4:274-81.

126. Yilmaz A, Rosch S, Klingel K, Kandolf R, Helluy X, Hiller KH, et al. Magnetic resonance imaging (MRI) of inflamed myocardium using iron oxide nanoparticles in patients with acute myocardial infarction - preliminary results. Int J Cardiol 2013;163:175-82.

127. Yilmaz A, Dengler MA, van der Kuip H, Yildiz H, Rosch S, Klumpp S, et al. Imaging of myocardial infarction using ultrasmall superparamagnetic iron oxide nanoparticles: a human study using a multi-parametric cardiovascular magnetic resonance imaging approach. Eur Heart J 2013;34:462-75.

128. Alam SR, Shah AS, Richards J, Lang NN, Barnes G, Joshi N, et al. Ultrasmall superparamagnetic particles of iron oxide in patients with acute myocardial infarction: early clinical experience. Circ Cardiovasc Imaging 2012;5:559-65.

129. Wilson SR, Sabatine MS, Braunwald E, Sloan S, Murphy SA, Morrow DA. Detection of myocardial injury in patients with unstable angina using a novel nanoparticle cardiac troponin I assay: observations from the PROTECT-TIMI 30 Trial. Am Heart J 2009;158:386-91.

130. Tang TY, Howarth SP, Miller SR, Graves MJ, Patterson AJ, UKing-Im JM, et al. The ATHEROMA (Atorvastatin Therapy: Effects on Reduction of Macrophage Activity) Study. Evaluation using ultrasmall superparamagnetic iron oxide-enhanced magnetic resonance imaging in carotid disease. J Am Coll Cardiol 2009;53:2039-50.

131. Degnan AJ, Patterson AJ, Tang TY, Howarth SP, Gillard JH. Evaluation of ultrasmall superparamagnetic iron oxide-enhanced MRI of carotid atherosclerosis to assess risk of cerebrovascular and cardiovascular events: follow-up of the ATHEROMA trial. Cerebrovasc Dis 2012;34:169-73.

132. Richards JM, Shaw CA, Lang NN, Williams MC, Semple SI, MacGillivray TJ, et al. In vivo mononuclear cell tracking using superparamagnetic particles of iron oxide: feasibility and safety in humans. Circ Cardiovasc Imaging 2012;5:509-17.

133. Chu-fan L, Xing W, Xun H, Gui-fu W, Cheng-heng H, Zhi-min D. Protective effect of lipid microspheres 1 on myocardial injury following elective percutaneous coronary intervention in patients with angina pectoris: a pilot study. J Cardiovasc Med (Hagerstown) 2011;12:790-4.

134. Winter PM, Caruthers SD, Zhang H, Williams TA, Wickline SA, Lanza GM. Antiangiogenic synergism of integrin-targeted fumagillin nanoparticles and atorvastatin in atherosclerosis. JACC Cardiovasc Imaging 2008;1:624-34.

135. Margolis J, McDonald J, Heuser R, Klinke P, Waksman R, Virmani R, et al. Systemic nanoparticle paclitaxel (nab-paclitaxel) for in-stent restenosis I (SNAPIST-I): a first-in-human safety and dose-finding study. Clin Cardiol 2007;30:165-70.

136. Tang TY, Muller KH, Graves MJ, Li ZY, Walsh SR, Young V, et al. Iron oxide particles for atheroma imaging. Arterioscler Thromb Vasc Biol 2009;29:1001-8.

137. Howarth SP, Tang TY, Trivedi R, Weerakkody R, U-King-Im JM, Gaunt ME, et al. Utility of USPIO-enhanced MR imaging to identify inflammation and the fibrous cap: a comparison of symptomatic and asymptomatic individuals. Eur J Radiol 2009;70:555-60.

138. Neuwelt EA, Hamilton BE, Varallyay CG, Rooney WR, Edelman RD, Jacobs PM, et al. Ultrasmall superparamagnetic iron oxides (USPIOs): a future alternative magnetic resonance (MR) contrast agent for patients at risk for nephrogenic systemic fibrosis (NSF)? Kidney Int 2009;75:465-74.

139. Soenen SJ, De Cuyper M. Assessing iron oxide nanoparticle toxicity in vitro: current status and future prospects. Nanomedicine (Lond) 2010;5:1261-75.

140. Hasebroock KM, Serkova NJ. Toxicity of MRI and CT contrast agents. Expert Opin Drug Metab Toxicol 2009;5: 403-16.

141. Bernd H, De Kerviler E, Gaillard S, Bonnemain B. Safety and tolerability of ultrasmall superparamagnetic iron oxide contrast agent: comprehensive analysis of a clinical development program. Invest Radiol 2009;44:336-42.

142. Winer JL, Liu CY, Apuzzo ML. The use of nanoparticles as contrast media in neuroimaging: a statement on toxicity. World Neurosurg 2012;78:709-11.

143. Desai N. Challenges in development of nanoparticle-based therapeutics. AAPS J 2012;14:282-95.

144. Asharani PV, Lianwu Y, Gong Z, Valiyaveettil S. Comparison of the toxicity of silver, gold and platinum nanoparticles in developing zebrafish embryos. Nanotoxicology 2011;5: 43-54.

145. Bar-Ilan O, Albrecht RM, Fako VE, Furgeson DY. Toxicity assessments of multisized gold and silver nanoparticles in zebrafish embryos. Small 2009;5:1897-910.

146. Van der Linden P, Ickx BE. The effects of colloid solutions on hemostasis. Can J Anaesth 2006;53(6 Suppl):S30-9.

147. Disa JJ, Polvora VP, Pusic AL, Singh B, Cordeiro PG. Dextranrelated complications in head and neck microsurgery: do the benefits outweigh the risks? A prospective randomized analysis. Plast Reconstr Surg 2003;112:1534-9.

148. Bhatt AP, Neppalli VT, Kelley EA, Schlueter AJ, Thomas CP. Dextran removal by plasmapheresis in a kidney-pancreas transplant recipient with dextran 40-induced osmotic nephrosis. Am J Kidney Dis 2011;57:621-3.

149. Dokka S, Toledo D, Shi X, Castranova V, Rojanasakul Y. Oxygen radical-mediated pulmonary toxicity induced by some cationic liposomes. Pharm Res 2000;17:521-5.

150. Hall JB, Dobrovolskaia MA, Patri AK, McNeil SE. Characterization of nanoparticles for therapeutics. Nanomedicine (Lond) 2007;2:789-803.

151. Keene AM, Peters D, Rouse R, Stewart S, Rosen ET, Tyner KM. Tissue and cellular distribution of gold nanoparticles varies based on aggregation/agglomeration status. Nanomedicine (Lond) 2012;7:199-209.

152. Oberdorster G. Safety assessment for nanotechnology and nanomedicine: concepts of nanotoxicology. J Intern Med 2010;267:89-105.

153. Owens DE, Peppas NA. Opsonization, biodistribution, and pharmacokinetics of polymeric nanoparticles. Int J Pharm 2006;307:93-102.

154. Kumar A, Dhawan A. Genotoxic and carcinogenic potential of engineered nanoparticles: an update. Arch Toxicol 2013;87:1883-900. 
155. Zolnik BS, Gonzalez-Fernandez A, Sadrieh N, Dobrovolskaia MA. Nanoparticles and the immune system. Endocrinology 2010;151:458-65.

156. Aillon KL, Xie Y, El-Gendy N, Berkland CJ, Forrest ML. Effects of nanomaterial physicochemical properties on in vivo toxicity. Adv Drug Deliv Rev 2009;61:457-66.

157. Lv H, Zhang S, Wang B, Cui S, Yan J. Toxicity of cationic lipids and cationic polymers in gene delivery. J Control Release 2006;114:100-9.

158. Yamashita K, Yoshioka Y, Higashisaka K, Mimura K, Morishita Y, Nozaki M, et al. Silica and titanium dioxide nanoparticles cause pregnancy complications in mice. Nat Nanotechnol 2011;6:321-8.

159. Buyukhatipoglu K, Miller TA, Clyne AM. Flame synthesis and in vitro biocompatibility assessment of superparamagnetic iron oxide nanoparticles: cellular uptake, toxicity and proliferation studies. J Nanosci Nanotechnol 2009;9:6834-43.

160. Dobrovolskaia MA, Neun BW, Clogston JD, Grossman JH, McNeil SE. Choice of method for endotoxin detection depends on nanoformulation. Nanomedicine (Lond) 2013. Dec. 20 [Epub ahead of print].

161. Kucki M, Cavelius C, Kraegeloh A. Interference of silica nanoparticles with the traditional Limulus amebocyte lysate gel clot assay. Innate Immun 2014;20:327-36.

162. Grainger DW. Nanotoxicity assessment: all small talk? Adv Drug Deliv Rev 2009;61:419-21.

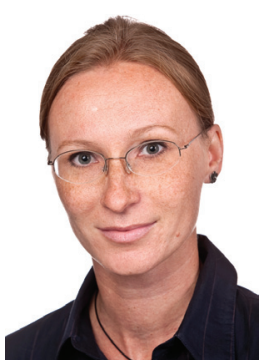

Iwona Cicha studied Biology at the Jagiellonian University, Cracow, Poland. After obtaining her PhD in medical sciences at the Ehime Medical School, Ehime University, Japan, she moved to the University of Erlangen, Germany. She was a postdoctoral fellow in the Department of Nephrology in 2003, before joining the Department of Cardiology, where she obtained her habilitation in Experimental Medicine in 2012. She has an extensive research experience in the field of atherosclerosis, with focus on the role of inflammation and blood flow dynamics in plaque development and destabilization. Since July 2013, she has been leading the Cardiovascular Nanomedicine Unit at the Section of Experimental Oncology and Nanomedicine (SEON), University Hospital Erlangen, focusing on the projects involving the application of nanomedical strategies for the treatment of cardiovascular disease.
163. Zolnik BS, Sadrieh N. Regulatory perspective on the importance of ADME assessment of nanoscale material containing drugs. Adv Drug Deliv Rev 2009;61:422-7.

164. Wacker M. Nanocarriers for intravenous injection - the long hard road to the market. Int J Pharm 2013;457:50-62.

165. Mei L, Zhang Z, Zhao L, Huang L, Yang XL, Tang J, et al. Pharmaceutical nanotechnology for oral delivery of anticancer drugs. Adv Drug Deliv Rev 2013;65:880-90.

166. Xie X, Tao Q, Zou Y, Zhang F, Guo M, Wang Y, et al. PLGA nanoparticles improve the oral bioavailability of curcumin in rats: characterizations and mechanisms. J Agric Food Chem 2011;59:9280-9.

167. Shaikh J, Ankola DD, Beniwal V, Singh D, Kumar MN. Nanoparticle encapsulation improves oral bioavailability of curcumin by at least 9 -fold when compared to curcumin administered with piperine as absorption enhancer. Eur J Pharm Sci 2009;37:223-30.

168. Paliwal R, Paliwal SR, Agrawal GP, Vyas SP. Biomimetic solid lipid nanoparticles for oral bioavailability enhancement of low molecular weight heparin and its lipid conjugates: in vitro and in vivo evaluation. Mol Pharm 2011;8:1314-21.

169. Jiao Y, Ubrich N, Marchand-Arvier M, Vigneron C, Hoffman $M$, Lecompte T, et al. In vitro and in vivo evaluation of oral heparin-loaded polymeric nanoparticles in rabbits. Circulation 2002;105:230-5.

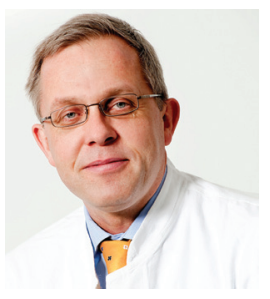

Christoph D. Garlichs, an experienced clinician specialized in interventional and experimental cardiology, studied Medicine and Philosophy in Berlin, Germany, and completed his doctoral thesis in 1996. He headed the Laboratories of Molecular Cardiovascular Research at the University of Dresden before becoming the Head of the Laboratory of Molecular Cardiology at the University of Erlangen-Nuremberg in 1998. He obtained his habilitation in Internal Medicine in 2002 and became Professor of Cardiology in 2008. Between 2007 and 2013 he was a Vice-Director in the Medical Clinic 2 (Cardiology, Angiology) of the University Hospital Erlangen. Since June 2013, he has been the head of the Internal Medicine Clinic at the DIAKO Hospital Flensburg, Germany, assuming clinical responsibility for cardiology, angiology, nephrology, and internal intensive care medicine. 


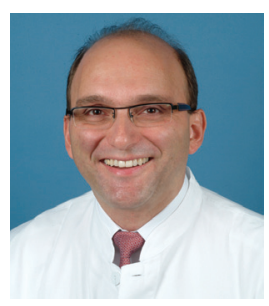

Christoph Alexiou received his PhD in 1995 from the Medical School of the Technical University of Munich, Germany. After the internship in the Gastroenterology Department, he worked as physician and researcher at the Department of Otorhinolaryngology, Head and Neck Surgery of the Technical University and founded a research group focusing on local chemotherapy using magnetic nanoparticles (Magnetic Drug Targeting). In 2000, he received his degree as an ENT-Physician and in 2002 he moved to the ENT-Department in Erlangen, Germany, where he obtained his postdoctoral lecture qualification (Habilitation). $\mathrm{He}$ is an assistant medical director in the ENT-Clinic and leads the Section for Experimental Oncology and Nanomedicine (SEON). Since 2009, he holds the Else Kröner-Fresenius-Foundation-Professorship for Nanomedicine at the University Hospital Erlangen. His research focuses on Magnetic Drug Targeting and the application of magnetic nanoparticles into human trials. For his work, he received several national and international awards. 